QUARTERLY OF APPLIED MATHEMATICS

VOLUME LXIII, NUMBER 1

MARCH 2005, PAGES 156-200

S 0033-569X(05)00949-0

Article electronically published on February 1, 2005

\title{
A COMPUTATIONAL AND STATISTICAL FRAMEWORK FOR MULTIDIMENSIONAL DOMAIN ACOUSTOOPTIC MATERIAL INTERROGATION
}

\author{
BY
}

H. T. BANKS (Center for Research in Scientific Computation, North Carolina State University, Raleigh, N.C. 27695-8205)

AND

V. A. BOKIL (Center for Research in Scientific Computation, North Carolina State University, Raleigh, N.C. 27695-8205)

\begin{abstract}
We consider an electromagnetic interrogation technique in two and three dimensions for identifying the dielectric parameters (including the permittivity, the conductivity and the relaxation time) of a Debye medium. In this technique, a travelling acoustic pressure wave in the Debye medium is used as a virtual reflector for an interrogating microwave electromagnetic pulse that is generated in free space. The reflections of the microwave pulse from the air-Debye interface and from the acoustic pressure wave are recorded at a remote antenna. The data is used in an inverse problem to estimate the locally pressure dependent dielectric parameters of the Debye medium. We present a time domain formulation that is solved using finite differences (FDTD) in time and in space. Perfectly matched layer (PML) absorbing boundary conditions are used to absorb outgoing waves at the finite boundaries of the computational domain, preventing spurious reflections from reentering the domain.

Using the method of least squares for the parameter identification problem, we compare two different algorithms (the gradient based Levenberg-Marquardt method and the gradient free, simplex based Nelder-Mead method) in solving an inverse problem to calculate estimates for two or more dielectric parameters. Finally we use statistical error analysis to construct confidence intervals for all the presented estimates, thereby providing a probabilistic statement about the computational procedure with uncertainty aspects of estimates.
\end{abstract}

Received August 14, 2004.

2000 Mathematics Subject Classification. Primary 78M20, 78A25, 78A02, 78A46, 62F25.

Key words and phrases. Electromagnetic-acoustic interaction, Debye dielectric materials, pulsed antenna source microwaves, inverse problems, FDTD, statistical inference.

E-mail address: htbanks@ncsu.edu

E-mail address: vabokil@ncsu.edu 
1. Introduction. The study of transient electromagnetic waves in lossy dispersive dielectrics is of great interest due to numerous applications [AMP94, APM89]. These include microwave imaging for medical applications in which one seeks to investigate the internal structure of an object (the human body) by means of electromagnetic fields at microwave frequencies $(300 \mathrm{MHz}-30 \mathrm{GHz})$. This is done, for example, to detect cancer or other anomalies by studying changes in the dielectric properties (such as permittivity, conductivity, relaxation times, etc.) of tissues FMS03. One generates the electrical property distributions in the body (Microwave maps) with the hope that such properties of different bodily tissues are related to their physiological state. With such noninvasive interrogating techniques one can study properties and defects in biological tissues with very little discomfort to the subjects. Other potential applications for such interrogation techniques are nondestructive damage detection in aircraft and spacecraft where very high frequency electromagnetic pulses can be used to detect the location and width of cracks that may be present [BGW03]. Additional applications are found in mine, ordnance and camouflage surveillance, and subsurface and atmospheric environmental modelling [BBL00].

Another important technique employed in noninvasive interrogation of objects is the use of ultrasonic waves in industrial and medical applications. The interaction of electromagnetic and sound waves in matter has been widely studied in acoustooptics And67 Kor97. Brillouin first showed that electromagnetic and sound waves can interact in a medium and influence each others' propagation dynamics Bri60. In particular, the response of the atomic electrons in a material medium to the applied electric field results in a polarization of the material with a resulting index of refraction that is a function of the density in the material. Consequently, the material density fluctuations produced by a sound wave induce perturbations in the index of refraction. Thus an electromagnetic wave transmitted through the medium will be modulated by the sound wave, and scattering and reflection will occur. Conversely, a spatial variation of the electromagnetic field intensity and the corresponding electromagnetic stress lead to a volume force distribution in the medium. This is called electrostriction, and can lead to sound generation. This mutual interaction between light and sound may also lead to instabilities and wave amplification MI68.

The work in this paper is based on ideas underlying the techniques formulated in BBL00 and ABR02. In BBL00, the authors present an electromagnetic interrogation technique for general dispersive media backed by either a perfect conducting wall to reflect the electromagnetic waves, or by using standing acoustic waves as virtual reflectors. In ABR02, this work was extended to using travelling waves as acoustic reflectors. In both cases the techniques were demonstrated in one dimension by assuming plane electromagnetic waves impinging at normal incidence on slabs of dispersive media. A one dimensional setting with normally incident waves is not geometrically feasible in many applications. In [BB04a a specialized multidimensional version of the electromagnetic interrogation problem is presented for dispersive media backed by perfect conductor walls and the finite difference time domain (FDTD) method is utilized [Taf95, Taf98]. However, the object of interrogation may not be backed by perfect conductor walls in many important applications, and hence one seeks another type of interface which will reflect 


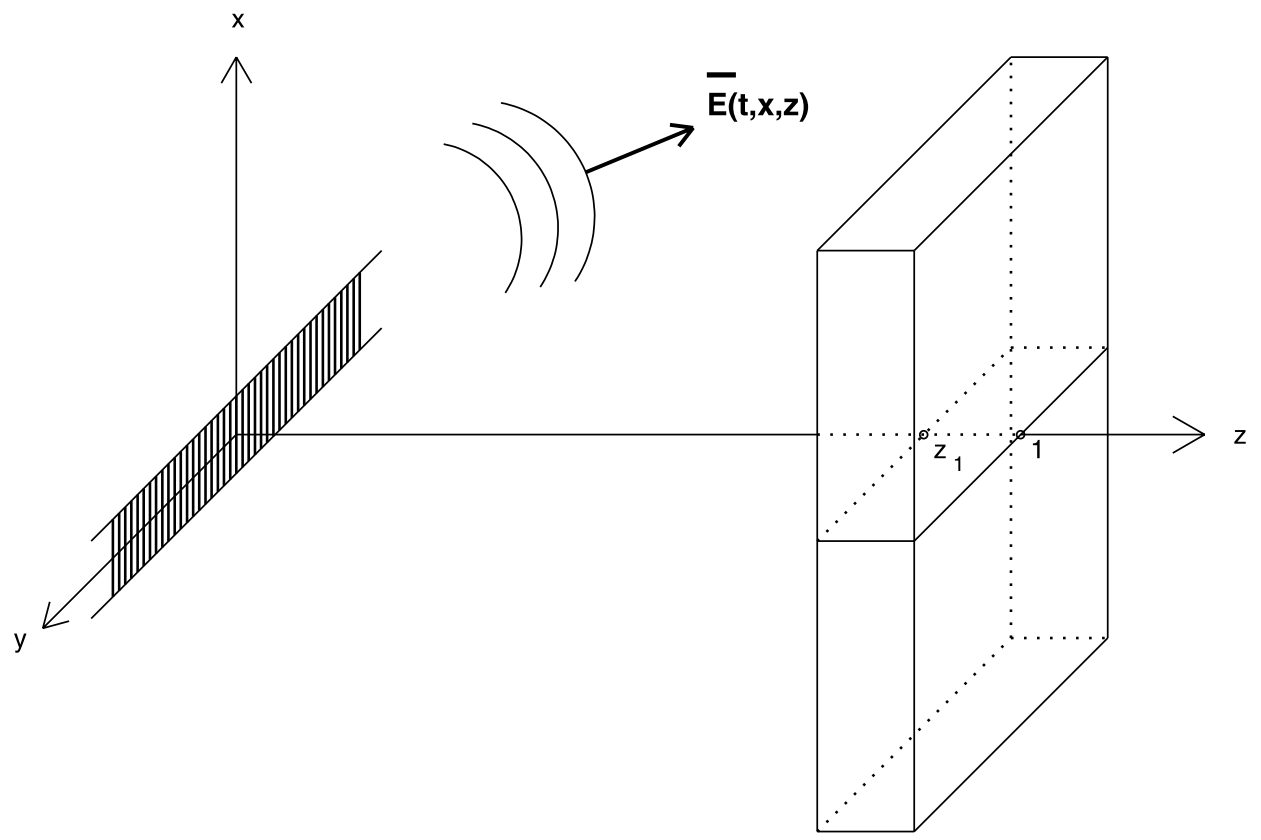

Fig. 1. Antenna and dielectric slab configuration.

the propagating electromagnetic pulses. It is therefore of great importance to ascertain (computationally and experimentally) whether the interrogation ideas of [ABR02] using acoustic pressure waves as virtual reflectors can be effective in multidimensional settings. The goal of this paper is the investigation of computational and statistical aspects of this methodology in higher dimensions. At present we are also trying to validate these proposed techniques by testing the ideas in this paper in an experimental setup. Our group has built an antenna that we are using to study the acoustooptical interaction in agar-agar in which the sound waves are produced by means of a transducer ABK04. In such a setup, as well as in many applications, one cannot assume normally incident plane waves impinging on the agar, and hence one needs to consider the more general case of oblique incidence. This further motivates the study in a multidimensional setting of an electromagnetic interrogation technique using the acoustooptical interaction to investigate dielectic properties of dispersive media.

We begin with Maxwell's equations for a first order dispersive (Debye) media with Ohmic conductivity and incorporate a model that describes the electromagnetic/acoustic interaction. Our model features modulation of the material polarization by the pressure wave and thus the behavior of the electromagnetic pulse. This approach is based on ideas found in [MI68]. We incorporate a pressure dependent Debye model for orientational polarization into Maxwell's equations. We model the propagation of a non-harmonic pulse from a finite antenna source in free space impinging obliquely on a planar interface into the Debye medium. We use the finite difference time domain method (FDTD) Taf95 to discretize Maxwell's equations and to compute the components of the electric 
field in the case where the signal and the dielectric parameters are independent of the $y$ variable. Figure 1 depicts the Debye dielectric slab geometry and the antenna that we model in our problem with the infinite dielectric slab perpendicular to the $z$-axis, uniform in the $y$-direction lying in $z_{1} \leq z \leq 1$, and the antenna finite in the $x$-direction lying in the region $-\infty \leq y \leq \infty, x_{1} \leq x \leq x_{2}$, respectively. An alternating current along the $x$ direction of the antenna located at $z=z_{c}$ then produces an electric field that is uniform in $y$ with nontrivial components $E_{x}$ and $E_{z}$ depending on $(t, x, z)$. When propagated in the $x z$-plane, this results in oblique incident waves on the dielectric surface in the $x y$-plane at $z=z_{1}$. (In BF95] the authors use Fourier-series in the frequency domain to compute the propagation of a time harmonic pulse train of plane waves that enter a dielectric across a planar boundary at oblique incidence. They showed that precursors are excited by short rise time pulses at oblique incidence. However, the use of Fourier series restricts this approach to harmonic pulses.) The oblique waves impinge on the planar interface that separates air and the Debye medium at $z=z_{1}$ where they undergo partial transmission and partial reflection. A reflected wave travels back to the antenna at $z=z_{c}$ and is recorded. The transmitted part of the wave travels through the Debye medium and intercepts an acoustic pressure wave that is generated in the dielectric medium. Here the microwave pulse undergoes partial transmission and reflection. The reflected wave travels back through the Debye medium, undergoes a secondary reflection/transmission at the air-Debye interface and is then recorded at the antenna. Thus the antenna records three pieces of information; the input source, the reflection of the electromagnetic pulse from the air-Debye interface and the reflection from the pressure wave region. This data is recorded at the center of the antenna and will be used in an inverse problem to estimate the dielectric properties of the Debye medium. We note that the acoustic wave speed is many orders of magnitude smaller than the speed of light. Thus the acoustic pressure wave is almost stationary relative to the microwave pulse. In this paper, we have assumed that the acoustic pressure wave is specified a priori and hence we assume that the pressure wave is not modified by the electromagnetic wave. This leads to a much simplified electromagnetic/acoustic interaction model. The dynamic interaction between the two and the appropriateness of this simplifying assumption are currently being investigated and will be the topic of a future paper.

An important computational issue in modelling two or three dimensional wave propagation is the construction of a finite computational domain and appropriate boundary conditions for terminating this domain in order to numerically simulate an essentially unbounded domain problem. We surround the domain of interest by perfectly matched absorbing layers (PML's), thus producing a finite computational domain. The original PML formulation (called the split field PML) is due to J. P. Berenger Ber94 and was based on a split form of Maxwell's equations in which all the fields themselves were split into orthogonal subcomponents. Many different variations of the PML are now available in the literature. Most of these different formulations, though equivalent to the split field PML, do not involve the splitting of the fields or splitting of Maxwell's equations. The PML formulation that we use in this paper is based on the anisotropic (uniaxial) formulation that was first proposed by Sacks et. al. [SKLL95]. 
We discuss numerical results for the forward problem for two different test cases. The relaxation times of the Debye media in these two test cases differ by many orders of magnitude. We first perform a sensitivity analysis in each test case to determine which parameters are most likely to be accurately estimated in an inverse problem. We also determine which of the pressure dependent parameters are the most significant in each of the test cases. As we shall see, the significant difference in the orders of magnitude of the relaxation times for the two test cases produces inverse problems with very different estimation properties.

We next discuss a parameter estimation problem in which we estimate model parameters for the two different Debye media from simulated data. We formulate our inverse problem using the method of least squares. We compare two different algorithms in solving the parameter estimation problems; the Levenberg-Marquardt method, which is a gradient based algorithm, and the Nelder-Mead method, which is based on simplices and is gradient free. For the first test case, these two algorithms produce similar results. However, in the second test case, we see the presence of many local minima and the Nelder-Mead algorithm converges to a local minima instead of the global minimum.

We also consider uncertainty in estimates due to data uncertainty. We present a model for generating noisy data that we use in our inverse problems to simulate experimental settings in which noise enters into the problem in a natural fashion. We then discuss a statistical error methodology to analyze the results of the inverse problems based on noisy data. This statistical error analysis is used to obtain confidence intervals for all the estimated parameters, thereby providing levels of confidence that can be associated with estimates obtained with our methodology.

2. Model formulation: The forward problem. We consider Maxwell's equations which govern the electric field $\mathbf{E}$ and the magnetic field $\mathbf{H}$ in a domain $\Omega$ with charge density $\rho$. Thus we consider the system

$$
\left\{\begin{array}{l}
\text { (i) } \frac{\partial \mathbf{D}}{\partial t}+\mathbf{J}-\nabla \times \mathbf{H}=0, \text { in }(0, T) \times \Omega, \\
\text { (ii) } \frac{\partial \mathbf{B}}{\partial t}+\nabla \times \mathbf{E}=0, \text { in }(0, T) \times \Omega, \\
\text { (iii) } \nabla \cdot \mathbf{D}=\rho, \text { in }(0, T) \times \Omega, \\
\text { (iv) } \nabla \cdot \mathbf{B}=0, \text { in }(0, T) \times \Omega, \\
\text { (v) } \mathbf{E} \times \mathbf{n}=0, \text { on }(0, T) \times \partial \Omega, \\
\text { (vi) } \mathbf{E}(0, \mathbf{x})=0, \quad \mathbf{H}(0, \mathbf{x})=0, \text { in } \Omega .
\end{array}\right.
$$

The fields $\mathbf{D}, \mathbf{B}$ are the electric and magnetic flux densities respectively. All the fields in (11) are functions of position $\mathbf{r}=(x, y, z)$ and time $t$. We have $\mathbf{J}=\mathbf{J}_{c}+\mathbf{J}_{s}$, where $\mathbf{J}_{c}$ is a conduction current density and $\mathbf{J}_{s}$ is the source current density. We assume only free space (actually the antenna in our example) can have a source current, and $\mathbf{J}_{c}$ is only found in the dielectric material. The condition $(\mathbb{1} \mathbf{v})$, where $\mathbf{n}$ is the unit outward normal to $\partial \Omega$, is a perfectly conducting boundary condition on the computational domain which 
we will replace with absorbing conditions in the sequel. With perfect conductor conditions, the computational boundary acts like a hard wall to impinging electromagnetic waves causing spurious reflections that would contaminate the data that we wish to use in our inverse problems.

Constitutive relations that relate the electric and magnetic fluxes $\mathbf{D}, \mathbf{B}$ and the electric current $\mathbf{J}_{\mathbf{c}}$ to the electric and magnetic fields $\mathbf{E}, \mathbf{H}$ are added to these equations to make the system fully determined and to describe the response of a material to the electromagnetic fields. In free space, these constitutive relations are $\mathbf{D}=\epsilon_{0} \mathbf{E}$, and $\mathbf{B}=\mu_{0} \mathbf{H}$, where $\epsilon_{0}$ and $\mu_{0}$ are the permittivity and the permeability of free space, respectively, and are constant [Jac99]. In general there are different possible forms for these constitutive relationships. In a frequency domain formulation of Maxwell's equations, these are usually converted to linear relationships between the dependent and independent quantities with frequency dependent coefficient parameters. We will consider the case of a dielectric in which magnetic effects are negligible, and we assume that Ohm's law governs the electric conductivity. Thus, within the dielectric medium we have constitutive relations that relate the flux densities $\mathbf{D}, \mathbf{B}$ to the electric and magnetic fields, respectively. We have

$$
\left(\begin{array}{ccl}
\text { (i) } \quad \mathbf{D} & =\epsilon_{0} \mathbf{E}+\mathbf{P} I_{D}, \\
\text { (ii) } \quad \mathbf{B} & =\mu_{0} \mathbf{H}, \\
\text { (iii) } & \mathbf{J}_{c} & =\sigma \mathbf{E} I_{D} .
\end{array}\right)
$$

In (2), $I_{D}$ denotes the indicator function on the Debye medium. Thus, $\mathbf{J}_{c}=0$ in air. The quantity $\mathbf{P}$ is called the electric polarization. Its contribution to the electric flux is zero in air and is nonzero in the dielectric. Electric polarization may be defined as the electric field induced disturbance of the charge distribution in a region. This polarization may have an instantaneous component as well as ones that do not occur instantaneously; the latter usually have associated time constants called the relaxation times, which are denoted by $\tau$. We let the instantaneous component of the polarization to be related to the electric field by means of a dielectric constant $\epsilon_{0} \chi$ and denote the remainder of the electric polarization as $\mathbf{P}_{\mathrm{R}}$. We have

$$
\mathbf{P}=\mathbf{P}_{\mathrm{I}}+\mathbf{P}_{\mathrm{R}}=\epsilon_{0} \chi \mathbf{E}+\mathbf{P}_{\mathrm{R}},
$$

and hence the constitutive law (2, i) becomes

$$
\mathbf{D}=\epsilon_{0} \epsilon_{r} \mathbf{E}+\mathbf{P}_{\mathrm{R}},
$$

where $\epsilon_{r}=(1+\chi)$ is the relative permittivity of the dielectric medium.

To describe the behavior of the media's macroscopic electric polarization $\mathbf{P}_{\mathrm{R}}$, we employ a general integral equation model in which the polarization explicitly depends on the past history of the electric field. This model is sufficiently general to include microscopic polarization mechanisms such as dipole or orientational polarization (Debye), as well as ionic and electronic polarization (Lorentz), and other frequency dependent polarization mechanisms And67 as well as multiples of such mechanisms represented by a distribution of the associated time constants (e.g., see [BG04). The resulting 
constitutive law can be given in terms of a polarization or displacement susceptibility kernel $g$ as

$$
\mathbf{P}_{\mathrm{R}}(t, x, z)=\int_{0}^{t} g(t-s, x, z) \mathbf{E}(s, x, z) d s,
$$

which, in the case (Debye) of interest to us here, can be written as

$$
g(t)=\mathrm{e}^{-t / \tau} \frac{\epsilon_{0}\left(\epsilon_{s}-\epsilon_{\infty}\right)}{\tau} .
$$

Such a Debye model can be represented in differential form as

$$
\begin{array}{r}
\tau \dot{\mathbf{P}}_{\mathrm{R}}+\mathbf{P}_{\mathrm{R}}=\epsilon_{0}\left(\epsilon_{s}-\epsilon_{\infty}\right) \mathbf{E} \\
\mathbf{D}=\epsilon_{0} \epsilon_{\infty} \mathbf{E}+\mathbf{P}_{\mathrm{R}},
\end{array}
$$

inside the dielectric, whereas $\mathbf{P}_{\mathrm{R}}=0, \epsilon_{\infty}=1$ in air, and $\epsilon_{\infty}=\epsilon_{r}$ inside the dielectric. We will henceforth denote $\mathbf{P}_{\mathrm{R}}$ by $\mathbf{P}$. In equations (5) and (7), the parameters $\epsilon_{s}$ and $\epsilon_{\infty}$ denote the static relative permittivity and the value of permittivity for an extremely high $(\approx \infty)$ frequency field, respectively. The constant $\epsilon_{\infty}$ is called the infinite frequency permittivity. Biological cells and tissues display very high values of the dielectric constants at low frequencies, and these values decrease in almost distinct steps as the excitation frequency is increased. This frequency dependence of permittivity is called dielectric dispersion and permits identification and investigation of a number of completely different underlying mechanisms. This property of dielectric materials makes the problem of parameter identification an important as well as very useful topic for investigation.

We will modify system (11) and the constitutive laws (2) by performing a change of variables that renders the system in a form that is convenient for analysis and computation. From (1) i) we have

$$
\frac{\partial}{\partial t}\left(\mathbf{D}+\int_{0}^{t} \mathbf{J}_{c}(s, \mathbf{x}) \mathrm{d} s\right)-\nabla \times \mathbf{H}=-\mathbf{J}_{s}, \text { in }(0, T) \times \Omega .
$$

Next, we define the new variable

$$
\widetilde{\mathbf{D}}(t, \mathbf{x})=\mathbf{D}(t, \mathbf{x})+\int_{0}^{t} \mathbf{J}_{c}(s, \mathbf{x}) \mathrm{ds} .
$$

Using definition (91) in (8) and (11) we obtain the modified system

$$
\begin{aligned}
& \text { (i) } \frac{\partial \widetilde{\mathbf{D}}}{\partial t}-\nabla \times \mathbf{H}=-\mathbf{J}_{s} \text { in }(0, T) \times \Omega \\
& \text { (ii) } \frac{\partial \mathbf{B}}{\partial t}+\nabla \times \mathbf{E}=0 \text { in }(0, T) \times \Omega \\
& \text { (iii) } \nabla \cdot \widetilde{\mathbf{D}}=0 \text { in }(0, T) \times \Omega \\
& \text { (iv) } \nabla \cdot \mathbf{B}=0 \text { in }(0, T) \times \Omega \\
& \text { (v) } \mathbf{E} \times \mathbf{n}=0 \text { on }(0, T) \times \partial \Omega \\
& \text { (vi) } \mathbf{E}(0, \mathbf{x})=\mathbf{0}, \mathbf{H}(0, \mathbf{x})=\mathbf{0} \text { in } \Omega
\end{aligned}
$$


We will henceforth drop the ${ }^{\sim}$ symbol on $\mathbf{D}$. We note that equation (10 iii) follows from the continuity equation $\frac{\partial \rho}{\partial t}+\nabla \cdot \mathbf{J}=0$, the assumption that $\rho(0)=0$, and the assumption that $\nabla \cdot \mathbf{J}_{s}=0$ (in the sense of distributions). The modified constitutive laws (2) after substitution of Ohm's law and (9) are

$$
\left(\begin{array}{ll}
\text { (i) } & \mathbf{D}(t, \mathbf{x})=\epsilon_{0} \epsilon_{\infty}(\mathbf{x}) \mathbf{E}(t, \mathbf{x})+\mathbf{P}_{\mathrm{R}}+\int_{0}^{t} \sigma(\mathbf{x}) \mathbf{E}(s, \mathbf{x}) \mathrm{ds} \\
\text { (ii) } & \mathbf{B}=\mu_{0} \mathbf{H}
\end{array}\right.
$$

with $\mathbf{P}_{\mathrm{R}}$ the solution of

$$
\tau \dot{\mathbf{P}}_{\mathrm{R}}+\mathbf{P}_{\mathrm{R}}=\epsilon_{0}\left(\epsilon_{s}-\epsilon_{\infty}\right) \mathbf{E}
$$

3. An anisotropic perfectly matched layer absorbing medium. In order to obtain a finite computational domain we surround the region of interest with perfectly matched absorbing layers (PML's). These layers have been demonstrated to have many orders of magnitude better absorbing capabilities than many of the approximate absorbing boundary conditions [Taf98. In this section we will first construct PML's that can be matched to free space and then we will discuss how this construction can be generalized to the case of a Debye medium.

As the source current $\mathbf{J}_{s}$ is zero inside the PML regions we will neglect $\mathbf{J}_{s}$ in this section. We model the PMLs as lossy regions by adding artificial loss terms to Maxwell's equations. This approach is based on using anisotropic material properties to describe the absorbing layer [SKLL95, Ged96, Rap95]. Starting with Maxwell's equations in the time domain we will derive a PML model in the frequency domain and then transform the corresponding equations to the time domain by taking the inverse Fourier transforms of the frequency domain equations. We define the modified permeability and permittivity tensors

$$
\left\{\begin{array}{l}
{[\bar{\mu}]=[\mu]+\frac{\left[\sigma_{M}\right]}{\mathrm{j} \omega}} \\
{[\bar{\epsilon}]=[\epsilon]+\frac{\left[\sigma_{E}\right]}{\mathrm{j} \omega} .}
\end{array}\right.
$$

Using the definitions (13) we define two new constitutive laws

$$
\left(\begin{array}{l}
\hat{\mathbf{B}}_{\text {new }}=[\bar{\mu}] \hat{\mathbf{H}}, \\
\hat{\mathbf{D}}_{\text {new }}=[\bar{\epsilon}] \hat{\mathbf{E}},
\end{array}\right.
$$

where for every field vector $\mathbf{V}, \hat{\mathbf{V}}$ denotes its Fourier transform. From the constitutive laws (14) we can write Maxwell's equations in time-harmonic form as

$$
\left(\begin{array}{l}
\mathrm{j} \omega \hat{\mathbf{D}}_{\text {new }}=\nabla \times \hat{\mathbf{H}}, \\
\mathrm{j} \omega \hat{\mathbf{B}}_{\text {new }}=-\nabla \times \hat{\mathbf{E}}, \\
\nabla \cdot \hat{\mathbf{D}}_{\text {new }}=0, \\
\nabla \cdot \hat{\mathbf{B}}_{\text {new }}=0 .
\end{array}\right.
$$

The split field PML introduced by Berenger Ber94 is a hypothetical medium based on a mathematical model. In [MP95] Mittra and Pekel showed that Berenger's PML 
was equivalent to Maxwell's equations with a diagonally anisotropic tensor appearing in the constitutive relations for $\mathbf{D}$ and $\mathbf{B}$. For a single interface, the anisotropic medium is uniaxial and is composed of both the electric and magnetic permittivity tensors. This uniaxial PML (UPML) formulation performs as well as the original split field PML while avoiding the nonphysical field splitting. As will be shown below, by properly defining a general constitutive tensor $[S]$, we can use the UPML in the interior working volume as well as the absorbing layer. This tensor provides a lossless isotropic medium in the primary computation zone and individual UPML absorbers adjacent to the outer lattice boundary planes for attenuation of spurious wave reflections. The fields excited within the UPML are also plane wave in nature and satisfy Maxwell's curl equations. The derivation of the PML properties of the tensor constitutive laws is also done directly by Sacks, et al., in SKLL95 and by Gedney in Ged96. We follow the derivation by Sacks, et al., here. We begin by considering planar electromagnetic waves in free space incident upon a PML half space. Starting with the impedance matching assumption, i.e., the impedance of the layer must match that of free space: $\epsilon_{0}^{-1} \mu_{0}=[\bar{\epsilon}]^{-1}[\bar{\mu}]$, we have

$$
\frac{[\bar{\epsilon}]}{\epsilon_{0}}=\frac{[\bar{\mu}]}{\mu_{0}}=[S]=\operatorname{diag}\left\{a_{1}, a_{1}, a_{3}\right\} .
$$

Hence, the constitutive parameters inside the PML layer are $[\bar{\epsilon}]=\epsilon_{0}[S]$ and $[\bar{\mu}]=\mu_{0}[S]$, where $[S]$ is a diagonal tensor.

Using (14) and (16), we thus find the constitutive laws for the perfectly matched layer are

where the tensor $[S]$ is

$$
\left\{\begin{array}{l}
\hat{\mathbf{D}}_{\text {new }}=\epsilon_{0}[S] \hat{\mathbf{E}} \\
\hat{\mathbf{B}}_{\text {new }}=\mu_{0}[S] \hat{\mathbf{H}}
\end{array}\right.
$$

$$
[S]=\left[\begin{array}{ccc}
a^{-1} & 0 & 0 \\
0 & a & 0 \\
0 & 0 & a
\end{array}\right] .
$$

The perfectly matched layer is therefore characterized by the single complex number $a$.

REMARK 1. To match the anisotropic PML along a planar boundary to a dispersive isotropic half-space, we will need to modify the above construction for the phasor-domain constitutive relation

$$
\hat{\mathbf{D}}=\epsilon_{0} \epsilon_{r}^{*}(\omega) \hat{\mathbf{E}},
$$

where $\epsilon_{r}^{*}(\omega)$ is the frequency dependent relative permittivity of the Debye medium, which will be defined in a standard manner in (31) below.

3.1. Implementation of the uniaxial PML in three dimensions. To apply the perfectly matched layer to electromagnetic computations, we replace the half infinite layer by a layer of finite depth backed with a more conventional boundary condition, such as a perfect electric conductor (PEC). This truncation of the layer will lead to reflections generated at the PEC surface, which can propagate back through the layer to re-enter the computational region. In this case, the reflection coefficient $R$ is now a function of the angle of incidence $\theta$, the depth of the PML $\delta$, as well as the parameter $a$ in (18). Thus, this parameter $a$ for the PML is chosen in order for the attenuation of waves in the PML to be sufficient so that the waves striking the PEC surface are negligible in 


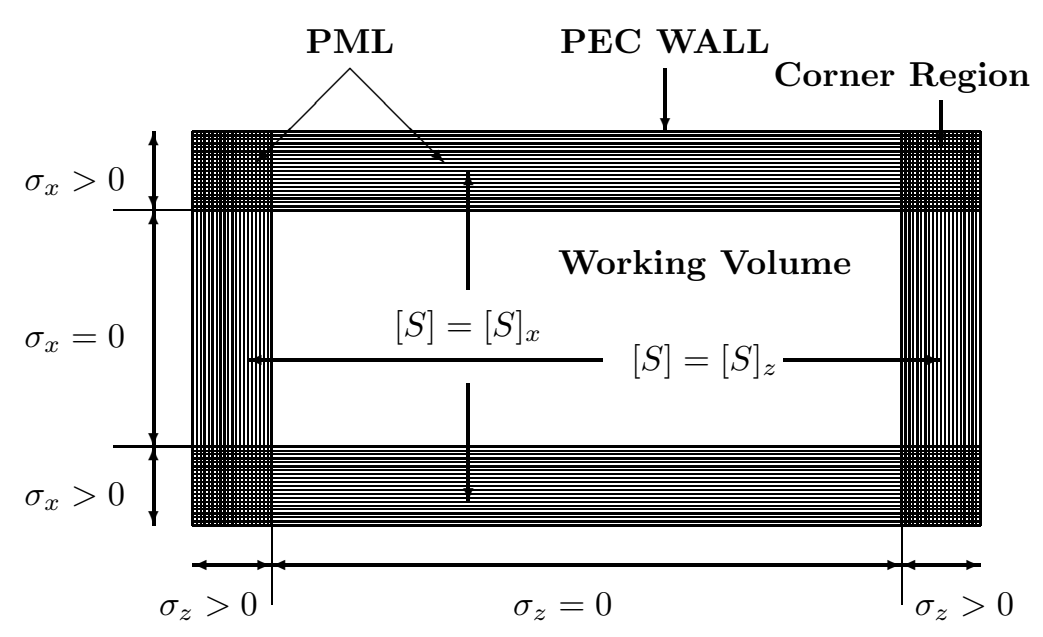

FIG. 2. PML layers surrounding the domain of interest. In the corner regions of the PML, both $\sigma_{x}$ and $\sigma_{z}$ are positive and the tensor [S] is the product $[S]_{x}[S]_{z}$. In the remaining regions only one of $\sigma_{z}$ (left and right PML's) or $\sigma_{x}$ (top and bottom PML's) are nonzero and positive. The tensor $[S]$ is thus either $[S]_{x}$ or $[S]_{z}$, respectively. The PML is truncated by a perfect electric conductor (PEC).

magnitude. Perfectly matched layers are then placed near each edge (or face in the 3D case) of the computational domain where a non-reflecting condition is desired. This leads to overlapping PML regions in the corners of the domain. As shown in [SKLL95], the correct form of the tensor that appears in the constitutive laws for these regions is the product

$$
[S]=[S]_{x}[S]_{y}[S]_{z},
$$

where component $[S]_{\alpha}$ in the product $(20)$ governs attenuation in the $\alpha$ direction, for $\alpha=x, y, z$ (see Figure 2). All three of the component tensors in (20) are diagonal and have the forms

$$
[S]_{x}=\left[\begin{array}{ccc}
s_{x}^{-1} & 0 & 0 \\
0 & s_{x} & 0 \\
0 & 0 & s_{x}
\end{array}\right] ;[S]_{y}=\left[\begin{array}{ccc}
s_{y} & 0 & 0 \\
0 & s_{y}^{-1} & 0 \\
0 & 0 & s_{y}
\end{array}\right] ;[S]_{z}=\left[\begin{array}{ccc}
s_{z} & 0 & 0 \\
0 & s_{z} & 0 \\
0 & 0 & s_{z}^{-1}
\end{array}\right]
$$

In the above, $s_{x}, s_{y}, s_{z}$ are analogous to the complex valued parameter $a$ encountered in the Section 3 analysis of the single PML layer.

When designing PMLs for implementation, it is important to choose the parameters $s_{\alpha}$ so that the resulting frequency domain equations can be easily converted back into 
$\Omega: \quad$ PEC wall

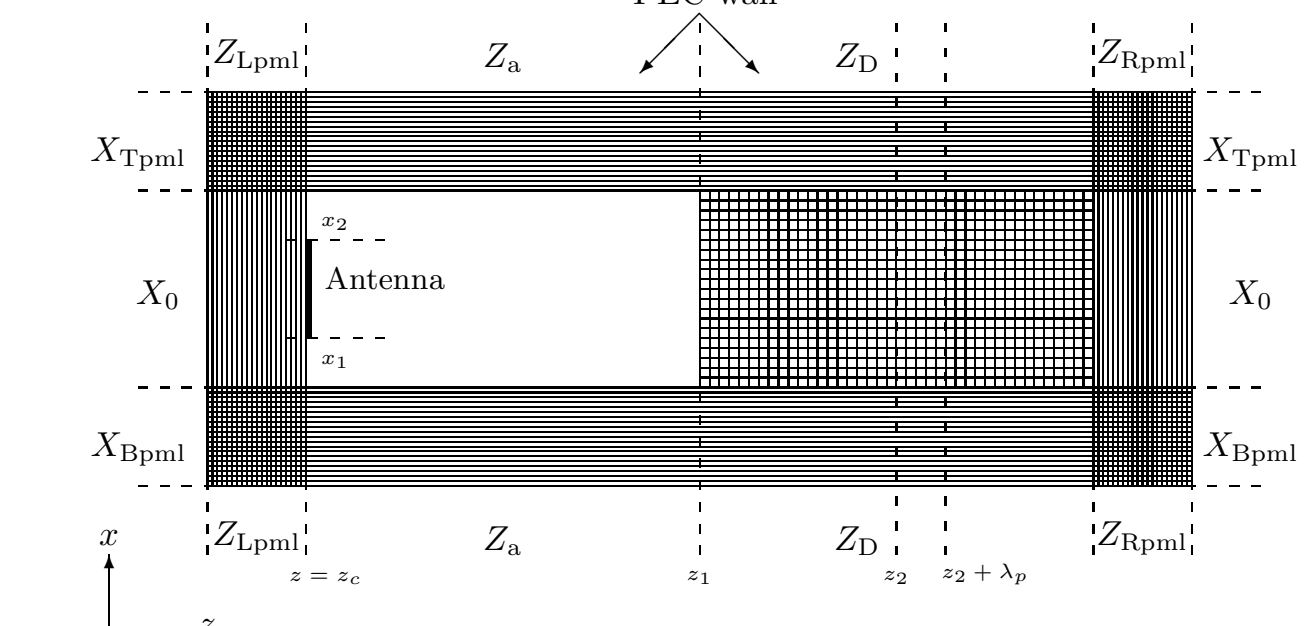

FIG. 3. The computational domain surrounded by PML layers.

the time domain. The simplest of these Ged96 which we employ here, is

$$
s_{\alpha}=1+\frac{\sigma_{\alpha}}{\mathrm{j} \omega \epsilon_{0}}, \quad \text { where } \quad \sigma_{\alpha} \geq 0, \quad \alpha=x, y, z .
$$

The PML interface represents a discontinuity in the conductivities $\sigma_{\alpha}$. To reduce the numerical reflections caused by these discontinuous conductivities, the $\sigma_{\alpha}$ are chosen to be functions of the variable $\alpha$ (e.g., $\sigma_{x}$ is taken to be a function of $x$ in the $[S]_{x}$ component of the PML tensor). A choice of these functions so that $\sigma_{\alpha}=0$, i.e., $s_{\alpha}=1$, at the interface yields the PML a continuous extension of the medium being matched and reduces numerical reflections at the interface. If one increases the value of $\sigma_{\alpha}$ with depth in the layer, one obtains greater overall attenuation while minimizing the numerical reflections. Gedney Ged96] suggests a conductivity profile

$$
\sigma_{\alpha}(\alpha)=\frac{\sigma_{\mathrm{opt}}\left|\alpha-\alpha_{0}\right|^{m}}{\delta^{m}} ; \quad \alpha=x, y, z,
$$

where $\delta$ is the depth of the layer, $\alpha=\alpha_{0}$ is the interface between the PML and the computational domain, and $m$ is the order of the polynomial variation. Gedney remarks that values of $m$ between 3 and 4 are believed to be optimal. Empirical testing suggests that, for a broad range of problems, an optimal value of $\sigma_{\max }$ is given by

$$
\sigma_{\mathrm{opt}} \approx \frac{m+1}{150 \pi \Delta_{\alpha \sqrt{\epsilon_{r}}}}
$$

where $\Delta_{\alpha}$ is the space increment in the $\alpha$ direction and $\epsilon_{r}$ is the relative permittivity of the material being modelled. In the case of free space $\epsilon_{r}=1$, since we desire to match the PML to both free space as well as the Debye medium, we will use $\epsilon_{r}$ to be the average value

$$
\epsilon_{r}=\frac{1}{2}\left(1+\epsilon_{\infty}\right)
$$


3.2. Reduction to two dimensions. From the time-harmonic Maxwell's curl equations in the PML (15) and (17), Faraday's and Ampere-Maxwell's laws can be written in the most general form as

$$
\left(\begin{array}{l}
\mathrm{j} \omega \mu_{0}[S] \hat{\mathbf{H}}=-\nabla \times \hat{\mathbf{E}}, \quad(\text { Faraday's Law) } \\
\mathrm{j} \omega[S] \hat{\mathbf{D}}=\nabla \times \hat{\mathbf{H}}-\mathbf{J}_{s}, \quad(\text { Ampere-Maxwell's Law) } .
\end{array}\right.
$$

In (26), $[S]$ is the diagonal tensor defined via (20), (21) and (22). In the presence of this diagonal tensor, a plane wave is purely transmitted into the uniaxial medium. The tensor $[S]$ is no longer uniaxial by strict definition, but rather is anisotropic. However, the anisotropic PML is still referenced as uniaxial since it is uniaxial in the non-overlapping PML regions. Let $\Omega=X \times Z$ denote the computational domain along with the absorbing layers. We partition the interval $X$ into disjoint closed intervals as $X=X_{\mathrm{Bpml}} \cup X_{0} \cup$ $X_{\text {Tpml }}$ and the interval $Z$ into disjoint closed intervals as $Z_{\mathrm{Lpml}} \cup Z_{\mathrm{a}} \cup Z_{\mathrm{D}} \cup Z_{\mathrm{Rpml}}$, as seen in Figure 3 The computational domain of interest is the region $X_{0} \times\left\{Z_{\mathrm{a}} \cup Z_{\mathrm{D}}\right\}$, where $X_{0} \times Z_{\mathrm{a}}$ denotes air and $X_{0} \times Z_{\mathrm{D}}$ denotes the Debye medium. The buffer region $\Omega \backslash\left(X_{0} \times\left\{Z_{\mathrm{a}} \cup Z_{\mathrm{D}}\right\}\right)$ contains the absorbing layers (PMLs). The PMLs are backed by a perfect conductor where the boundary condition $\mathbf{n} \times \mathbf{E}=0$ is used. To obtain a two-dimensional model, we make the assumption that the signal and the dielectric parameters are independent of the $y$ variable. Also, an alternating current along the $x$ direction, as in (45) below, then produces an electric field that is uniform in $y$ with nontrivial components $E_{x}$ and $E_{z}$ depending on $(t, x, z)$ which, when propagated in the $x z$ plane, result in oblique incident waves on the dielectric surface in the $x y$ plane at $z=z_{1}$. With these assumptions, Maxwell's equations reduce to a two dimensional system for solutions involving the $E_{x}$ and $E_{z}$ components for the electric field and the component $H_{y}$ for the magnetic field, which is referred to as the $\mathrm{TE}_{y}$ mode. In this case, we have $\sigma_{y}=0$ and $s_{y}=1$ in the UPML. From (11, i) and (7) expressed in the frequency domain, we have the constitutive relation

$$
\hat{\mathbf{D}}=\epsilon_{0}\left(\epsilon_{\infty}+\frac{\epsilon_{s}-\epsilon_{\infty}}{1+\mathrm{j} \omega \tau}+\frac{\sigma}{\mathrm{j} \omega \epsilon_{0}}\right) \hat{\mathbf{E}} .
$$

Re-scaling the electric field as

$$
\hat{\tilde{\mathbf{E}}}=\sqrt{\frac{\epsilon_{0}}{\mu_{0}}} \hat{\mathbf{E}},
$$

we can write the time-harmonic frequency domain Maxwell's equations (26) in the uniaxial medium as

$$
\left(\begin{array}{l}
\text { (i) } \mathrm{j} \omega\left(1+\frac{\sigma_{z}(z)}{\mathrm{j} \omega \epsilon_{0}}\right)\left(1+\frac{\sigma_{x}(x)}{\mathrm{j} \omega \epsilon_{0}}\right)^{-1} \hat{\tilde{D}}_{x}=-c_{0}\left(\frac{\partial \hat{H}_{y}}{\partial z}+\mathbf{J}_{s} \cdot \mathbf{i}\right), \\
\text { (ii) } \mathrm{j} \omega\left(1+\frac{\sigma_{x}(x)}{\mathrm{j} \omega \epsilon_{0}}\right)\left(1+\frac{\sigma_{z}(z)}{\mathrm{j} \omega \epsilon_{0}}\right)^{-1} \hat{\tilde{D}}_{z}=c_{0} \frac{\partial \hat{H}_{y}}{\partial x} \\
\text { (iii) } \mathrm{j} \omega\left(1+\frac{\sigma_{x}(x)}{\mathrm{j} \omega \epsilon_{0}}\right)\left(1+\frac{\sigma_{z}(z)}{\mathrm{j} \omega \epsilon_{0}}\right) \hat{H}_{y}=c_{0}\left(\frac{\partial \hat{E}_{z}}{\partial x}-\frac{\partial \hat{E}_{x}}{\partial z}\right) .
\end{array}\right.
$$


In the above, $\mathbf{i}$ is the unit vector in the $x$ direction, $c_{0}=3.0 \times 10^{8} \mathrm{~m} / \mathrm{s}$ is the speed of light in vacuum and $\hat{\tilde{\mathbf{D}}}=\left(\hat{\tilde{D}}_{x}, \hat{\tilde{D}}_{z}\right)^{T}$ is defined as

$$
\hat{\tilde{\mathbf{D}}}=\epsilon_{r}^{*} \cdot \hat{\tilde{\mathbf{E}}}
$$

with

$$
\epsilon_{r}^{*}=\epsilon_{\infty}+\frac{\epsilon_{s}-\epsilon_{\infty}}{1+j \omega \tau}+\frac{\sigma}{j \omega \epsilon_{0}} .
$$

To avoid a computationally intensive implementation, we define suitable constitutive relationships that facilitate the decoupling of the frequency dependent terms [Taf98. To this end, we introduce the fields

$$
\left(\begin{array}{l}
\hat{D}_{x}^{*}=s_{x}^{-1} \hat{\tilde{D}}_{x} \\
\hat{D}_{z}^{*}=s_{z}^{-1} \hat{\tilde{D}}_{z}, \\
\hat{H}_{y}^{*}=s_{z} \hat{H}_{y} .
\end{array}\right.
$$

Again, by dropping the ${ }^{\sim}$ symbol on $\mathbf{D}$ and transforming the frequency domain equations to the time domain, we obtain the system

$$
\left[\begin{array}{ll}
\text { (i) } & \partial_{t} H_{y}^{*}+\frac{\sigma_{x}(x)}{\epsilon_{0}} H_{y}^{*}=c_{0}\left(\frac{\partial E_{z}}{\partial x}-\frac{\partial E_{x}}{\partial z}\right) \\
\text { (ii) } & \partial_{t} H_{y}+\frac{\sigma_{z}(z)}{\epsilon_{0}} H_{y}=\partial_{t} H_{y}^{*} \\
\text { (iii) } & \partial_{t} D_{x}^{*}+\frac{\sigma_{z}(z)}{\epsilon_{0}} D_{x}^{*}=-c_{0}\left(\frac{\partial H_{y}}{\partial z}+\mathbf{J}_{s} \cdot \mathbf{i}\right) \\
\text { (iv) } & \partial_{t} D_{x}=\partial_{t} D_{x}^{*}+\frac{\sigma_{x}(x)}{\epsilon_{0}} D_{x}^{*} \\
\text { (v) } & \partial_{t} D_{z}^{*}+\frac{\sigma_{x}(x)}{\epsilon_{0}} D_{z}^{*}=c_{0} \frac{\partial H_{y}}{\partial x} \\
\text { (vi) } & \partial_{t} D_{z}=\partial_{t} D_{z}^{*}+\frac{\sigma_{z}(z)}{\epsilon_{0}} D_{z}^{*}
\end{array}\right.
$$

with

$$
\mathbf{D}(t)=\epsilon_{\infty} \mathbf{E}(t)+\mathbf{P}(t)+\mathbf{C}(t),
$$

where $\mathbf{D}=\left(D_{x}, D_{z}\right)^{T}$ and, inside of the dielectric, the polarization $\mathbf{P}=\left(P_{x}, P_{z}\right)^{T}$ satisfies

$$
\tau \dot{\mathbf{P}}+\mathbf{P}=\left(\epsilon_{s}-\epsilon_{\infty}\right) \mathbf{E},
$$

while the conductivity term $\mathbf{C}=\left(C_{x}, C_{y}\right)^{T}$ satisfies

$$
\dot{\mathbf{C}}=\left(\sigma / \epsilon_{0}\right) \mathbf{E}
$$

inside of the dielectric. Outside the dielectric we have $\mathbf{P}=\mathbf{C}=\mathbf{0}$. We will assume zero initial conditions for all the fields. 
3.3. Pressure dependence of polarization. We consider a pressure dependent Debye model for orientational polarization first developed in ABR02. We assume that the material dependent parameters in the differential equation (35) for the Debye medium depend on pressure $p$, i.e.,

$$
\tau(p) \dot{\mathbf{P}}+\mathbf{P}=\left(\epsilon_{s}(p)-\epsilon_{\infty}(p)\right) \mathbf{E} .
$$

We suppose as a first approximation that each of the pressure dependent parameters can be represented as a mean value plus a perturbation that is proportional to the pressure

$$
\left\{\begin{array}{l}
\text { (i) } \quad \tau(p)=\tau_{0}+\kappa_{\tau} p \\
\text { (ii) } \quad \epsilon_{s}(p)=\epsilon_{s, 0}+\kappa_{s} p \\
\text { (iii) } \quad \epsilon_{\infty}(p)=\epsilon_{\infty, 0}+\kappa_{\infty} p .
\end{array}\right.
$$

This can be considered as a truncation after the first-order terms of power series expansions of these functions of $p$. Thus (35) is written as

$$
\left(\tau_{0}+\kappa_{\tau} p\right) \dot{\mathbf{P}}+\mathbf{P}=\left(\left(\epsilon_{s, 0}-\epsilon_{\infty, 0}\right)+\left(\kappa_{s}-\kappa_{\infty}\right) p\right) \mathbf{E} .
$$

This model features modulation of the material polarization by the pressure wave and thus the behavior of the electromagnetic pulse. We will assume that the acoustic pressure wave is specified a priori. The pressure $p$ will be assumed to have the form

$$
p(t, z)=I_{\left(z_{2}, z_{2}+\lambda_{p}\right)} \sin \left(\omega_{p}\left[t+\frac{z-z_{2}}{c_{p}}\right]\right)
$$

where $z_{2} \in Z_{\mathrm{D}}$ with $z_{1}<z_{2}$. The terms $\omega_{p}, \lambda_{p}$ and $c_{p}$ denote the acoustic frequency, wavelength and speed, respectively. We note that outside the pressure region $\left(z_{2}, z_{2}+\lambda_{p}\right)$, the pressure is zero and

$$
\tau=\tau_{0}, \quad \epsilon_{s}=\epsilon_{s, 0}, \quad \epsilon_{\infty}=\epsilon_{\infty, 0}
$$

From (34),

$$
\left(\begin{array}{l}
\text { (i) } \quad D_{x}(t)=\left(\epsilon_{\infty, 0}+\kappa_{\infty} p\right) E_{x}(t)+P_{x}(t)+C_{x}(t), \\
\text { (ii) } \quad D_{z}(t)=\left(\epsilon_{\infty, 0}+\kappa_{\infty} p\right) E_{z}(t)+P_{z}(t)+C_{z}(t),
\end{array}\right.
$$

with

$$
\left(\begin{array}{ll}
\text { (i) } & \left(\tau_{0}+\kappa_{\tau} p\right) \dot{P}_{x}+P_{x}=\left(\left(\epsilon_{s, 0}-\epsilon_{\infty, 0}\right)+\left(\kappa_{s}-\kappa_{\infty}\right) p\right) E_{x} \\
\text { (ii) } & \left(\tau_{0}+\kappa_{\tau} p\right) \dot{P}_{z}+P_{z}=\left(\left(\epsilon_{s, 0}-\epsilon_{\infty, 0}\right)+\left(\kappa_{s}-\kappa_{\infty}\right) p\right) E_{z}
\end{array}\right.
$$

and

$$
\left(\begin{array}{ll}
\text { (i) } \quad \dot{C}_{x} & =\left(\sigma / \epsilon_{0}\right) E_{x} \\
\text { (ii) } & \dot{C}_{z}=\left(\sigma / \epsilon_{0}\right) E_{z}
\end{array}\right.
$$


3.4. Source term. The source term $\mathbf{J}_{s}$ will model an infinite (in the $y$ direction) antenna strip, finite in the $x$ direction (between $x_{1}$ and $x_{2}$ ), lying in the $z=z_{c}$ plane in free space, and we assume the signal is polarized with oscillations in the $x z$ plane only, with uniformity in the $y$ direction (see again Figure1). We therefore take

$$
\begin{aligned}
& \mathbf{J}_{s}(t, x, z)=I_{\left(x_{1}, x_{2}\right)}(x) \delta\left(z-z_{c}\right) \sin \left(\omega_{c}\left(t-3 t_{0}\right)\right) \exp \left(-\left[\frac{t-3 t_{0}}{t_{0}}\right]^{2}\right) \mathbf{i}, \\
& t_{0}=\frac{2}{\omega_{d}-\omega_{c}} \mathrm{sec}, \omega_{c}=2 \pi f_{c} \mathrm{rad} / \mathrm{sec} .
\end{aligned}
$$

Here $I_{\left(x_{1}, x_{2}\right)}$ is the indicator function on the interval $\left(x_{1}, x_{2}\right) \in X_{0}, \delta\left(z-z_{c}\right)$ is the Dirac measure centered at $z=z_{c}, \omega_{c}$ is the central angular frequency of the input signal and $\omega_{d}$ is the decay frequency. The Fourier spectrum of this pulse has even symmetry about the central frequency $f_{c}$. The pulse is centered at time step $3 t_{0}$ and has a $1 / e$ characteristic decay of $t_{0}$ time-steps [Taf95]. We will specify the values of all the different quantities involved in (45) when we discuss particular problems below. The source (45) radiates numerical waves having time waveforms corresponding to the source function. The radiated numerical wave will propagate to the Debye medium and undergo partial transmission and partial reflection, and the solution can be continued until all transients effectively decay.

3.5. Space and time discretization. We will use the FDTD algorithm [Taf95] to discretize Maxwell's equations. This algorithm uses forward differences to approximate the time and spatial derivatives. The FDTD technique rigorously enforces the vector field boundary conditions at interfaces of dissimilar media at the time scale of a small fraction of the impinging pulse width or carrier period. This approach is very general and permits accurate modelling of a broad variety of materials ranging from living human tissue to radar absorbers to optical glass.

The system of equations (33) can be discretized on the standard Yee lattice. The domain $\Omega$ is divided into square cells of length $h=\Delta x=\Delta z, h$ being the spatial increment. The electric field degrees of freedom are at the midpoints of edges of the squares and the magnetic degrees of freedom are at the centers of cells. The time interval over which time stepping is done is divided into subintervals of equal size using the time increment $\Delta t$. We perform normal leapfrogging in time and the loss terms are averaged in time. This leads to an explicit time stepping scheme (see BB04b for details). In this case, a stability condition (CFL) has to be satisfied [Taf95] in order to obtain a well posed computational scheme. The time step $\Delta t$ and the spatial increment $h$ for the FDTD scheme in non-dispersive dielectics are related by the condition

$$
\eta_{\mathrm{CN}}=\frac{c_{0} \Delta t}{h}<\frac{1}{\sqrt{2}}
$$

The number $\eta_{\mathrm{CN}}$ is called the Courant number. We fix the value of $\eta_{\mathrm{CN}}=1 / 2$. In [Pet94, the author established that several extensions of the FDTD for Debye media satisfy the stability restriction of the standard FDTD scheme in nondispersive dielectrics. He has also shown that the extensions do not preserve the non-dissipative character of the standard FDTD scheme. The extended difference systems are more dispersive than 
the standard FDTD and their accuracy depends strongly on how well the chosen timestep resolves the shortest timescale in the problem, regardless of whether it is the incident pulse timescale, the medium relaxation timescale, or the medium resonance timescale.

4. The forward problem for a Debye medium: First test case. As discussed in Section [3.3. the pressure dependent parameters $\epsilon_{s}, \epsilon_{\infty}$, and $\tau$ are represented as a mean value plus a perturbation that is proportional to the pressure in equations (38) (as discussed in ABR02]). In our first test case, we present numerical simulations for a Debye medium that is similar to water. For signal bandwidths in the microwave regime, the dispersive properties of pure water are usually modelled by a Debye equation having a single molecular relaxation term. The mean values $\epsilon_{s, 0}^{*}, \epsilon_{\infty, 0}^{*}$, and $\tau_{0}^{*}$ for this Debye model APM89 are given by

$$
\begin{aligned}
& \epsilon_{s, 0}^{*}=80.1 \quad \text { (relative static permittivity), } \\
& \epsilon_{\infty, 0}^{*}=5.5 \text { (relative high frequency permittivity) } \\
& \tau_{0}^{*} \quad=8.1 \times 10^{-12} \text { seconds }, \\
& \sigma^{*} \quad=1 \times 10^{-5} \text { mhos } / \text { meter }
\end{aligned}
$$

where the ${ }^{*}$ superscript will denote the true values of all the corresponding dielectric parameters. Since we do not yet have experimental data to determine the actual values, we instead choose trial values for the coefficients of pressure, $\kappa_{s}^{*}, \kappa_{\infty}^{*}$ and $\kappa_{\tau}^{*}$, to use in generating simulated data. As a first approximation we take these trial values to be a fraction of the mean values of the dielectric parameters, $\epsilon_{s, 0}^{*}, \epsilon_{\infty, 0}^{*}$, and $\tau_{0}^{*}$, respectively. Thus, we choose

$$
\begin{aligned}
\kappa_{s}^{*} & =0.6 \epsilon_{s, 0}^{*}=48.06, \\
\kappa_{\infty}^{*} & =0.8 \epsilon_{\infty, 0}^{*}=4.4, \\
\kappa_{\tau}^{*} & =0.05 \tau_{0}^{*}=4.05 \times 10^{-13} .
\end{aligned}
$$

We hope to determine the values of these pressure coefficients from appropriately designed experiments in the near future. We have assumed that the effect of the electric field on the acoustic pressure is negligible. We are interested in how and to what extent the acoustic pressure can change the reflected electric wave from the interface at $z=z_{2}$. Moreover, the relaxation parameter is a characteristic of the material in $\left[z_{1}, z_{2}\right]$, which affects the transmission of electric waves through this region. Consequently, we will examine in more detail how $\tau_{0}$ can affect the electric field. The electromagnetic input source has the form

$$
\begin{aligned}
\mathbf{J}_{s}(t, x, z) & =I_{\left(x_{1}, x_{2}\right)} \delta(z) \sin \left(\omega_{c}\left(t-3 t_{0}\right)\right) \exp \left(-\left[\frac{t-3 t_{0}}{t_{0}}\right]^{2}\right) \mathbf{i} \\
t_{0} & =\frac{1}{2 \pi \times 10^{9}} \mathrm{sec}, \omega_{c}=6 \pi \times 10^{9} \mathrm{rad} / \mathrm{sec}, f_{c}=3.0 \times 10^{9} \mathrm{~Hz} .
\end{aligned}
$$

The Fourier spectrum of this pulse has even symmetry about $3.0 \mathrm{GHz}$. The computational domain is defined as follows. We take $X_{0}=(0,0.1), Z_{\mathrm{a}}=(0,0.15)$ and $Z_{\mathrm{D}}=(0.15,0.2)$. The number of nodes along the $z$-axis is taken to be 320 and the 
number of nodes along the $x$-axis is taken to be 160 . The spatial step size in both the $x$ and $z$ directions is $\Delta x=\Delta z=h=0.1 / 160$. From the CFL condition (46) with the Courant number $\eta_{\mathrm{CN}}=1 / 2$, we obtain the time increment to be $\Delta t \approx 1.0417$ pico seconds. The central frequency of the input source as described in (49) is $3.0 \mathrm{GHz}$ and based on the speed of light in air, $c_{0}=3 \times 10^{8} \mathrm{~m} / \mathrm{s}$, we calculate the corresponding central wavelength to be $\lambda_{c}=\left(2 \pi c_{0}\right) / \omega=0.1$ meters. The antenna is half a wavelength long and is placed at $\left(x_{1}, x_{2}\right) \times z_{c}$, with $z_{c}=0, x_{1}=0.025$ and $x_{2}=0.075$. We use PML layers that are half a wavelength thick on all four sides of the computational domain as shown in Figure 3 The reflections of the electromagnetic pulse at the air-Debye interface and from the acoustic pressure wave are recorded at the center of the antenna $\left(x_{c}, z_{c}\right)$, with $x_{c}=0.05$, at every time step. This data will be used as observations for the parameter identification problem to be presented later. The component of the electric field that is of interest here is the $E_{x}$ component. Thus our data is the set $\mathbf{E}\left(\mathbf{q}^{*}\right)=\left\{E_{x}\left(n \Delta t, x_{c}, z_{c} ; \mathbf{q}^{*}\right)\right\}_{n=1}^{M}$, where $\mathbf{q}^{*}=\left(\epsilon_{s, 0}^{*}, \epsilon_{\infty, 0}^{*}, \tau_{0}^{*}, \sigma^{*}, \kappa_{s}^{*}, \kappa_{\infty}^{*}, \kappa_{\tau}^{*}\right)^{T}$. The windowed acoustic pressure wave as defined in (40) has the parameter values $\omega_{p}=6.0 \pi \times 10^{5}$ $\mathrm{rad} / \mathrm{sec}, c_{p}=1500 \mathrm{~m} / \mathrm{s}$, and thus, $\lambda_{p}=0.005 \mathrm{~m}$. The location of the pressure region is in the interval $\left(z_{2}, z_{2}+\lambda_{p}\right)=(0.175,0.18)$.

In Figure 4 (left) we plot the electromagnetic source that is used in the simulations for the Debye model (47). We plot the power spectral density of the source (49) in Figure 4 (right). The power spectral density $|Y|^{2}$ of the vector $\mathbf{J}_{s}$ is defined to be

$$
\begin{aligned}
\mathcal{Z} & =\operatorname{FFT}\left(\mathbf{J}_{s}\right), \\
|Y|^{2} & =\mathcal{Z} \cdot \overline{\mathcal{Z}},
\end{aligned}
$$

where $\operatorname{FFT}\left(\mathbf{J}_{s}\right)$ is the fast Fourier transform of the vector $\mathbf{J}_{\mathbf{s}}$. As seen in Figure 4 (right) the power spectral density is symmetric about $3.0 \mathrm{GHz}$.

In Figure 5 we plot the $E_{x}$ field magnitude at the center of the antenna versus time, which shows the electromagnetic source, the reflection off the air-Debye interface and the reflection from the region containing the acoustic pressure wave. As seen in these plots, the amplitude of the reflection from the acoustic pressure is many orders of magnitude smaller than that of the initial electromagnetic source as well as that of the reflection from the air-Debye interface. Thus, it is an interesting question as to whether the reflections from the acoustic pressure region can be used for identification of parameters.

In Figure 6] we plot the $E_{x}$ field magnitude at different times in the plane containing the center of the antenna versus depth along the $z$ axis. The top left figure shows the electromagnetic wave penetrating the Debye medium. In the top right figure we see the reflection of the electromagnetic wave from the Debye medium moving towards the antenna and the Brillouin precursor propagating in the Debye medium. In the bottom left figure we observe the reflection from the region containing the acoustic pressure and the transmitted part of the electromagnetic source travelling into the absorbing layer. The reflection from the acoustic pressure crosses the Debye medium in the bottom right figure. 

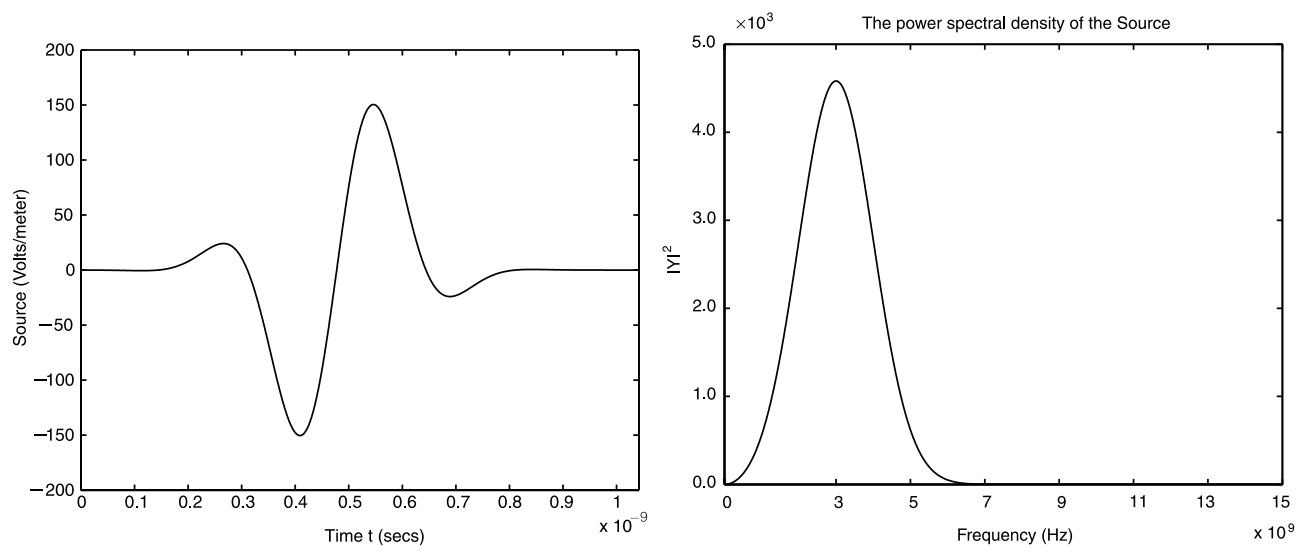

FIG. 4. Forward simulation for a Debye medium with parameters given in (47) and (48). (Left) The source as defined in (49). (Right) Fourier transform of the source. The transform is centered around the central angular frequency of $\omega_{c}=6 \pi \times 10^{9} \mathrm{rad} / \mathrm{sec}$.
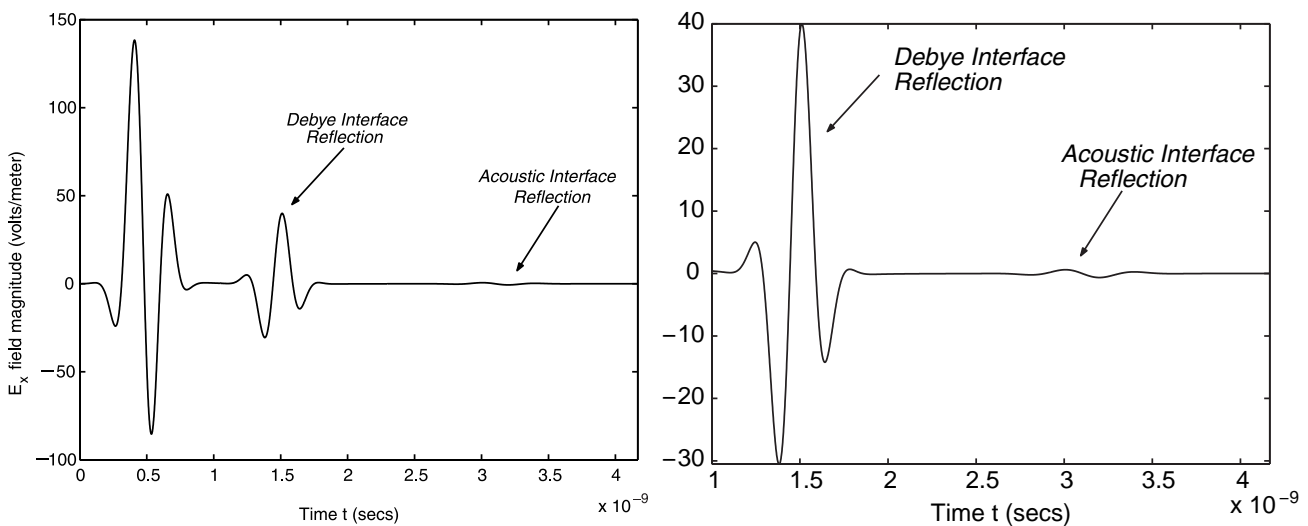

FIG. 5. Forward simulation for a Debye medium with parameters given in (47) and (48). Time plot of the $E_{x}$ component of the electric field. This is the "data" that is received at the center of the antenna. (Left) The original signal (source), the reflection off the Air-Debye interface and the reflection due to the pressure wave. (Right) The (scale enlarged) reflection off the Air-Debye interface and the reflection due to the pressure wave.

4.1. Sensitivity analysis. In this section we examine the system dynamics as the parameters vary. In particular, we are interested in the changes produced by the coefficients of pressure in the polarization, namely the parameters $\kappa_{s}, \kappa_{\infty}$ and $\kappa_{\tau}$.

We consider equation (31) to argue that the parameter $\kappa_{s}$ appears to be the most influential in the wave interaction, whereas the pressure coefficients $\kappa_{\infty}$ and $\kappa_{\tau}$ do not seem to be as important. In our simulations the outgoing and reflected radiation will be dominated by frequencies near the center frequency $3.0 \mathrm{GHz}$. Thus, $\epsilon_{r}^{*}$ will be dominated by frequencies near $3.0 \mathrm{GHz}$. In this problem $\omega \tau_{0}^{*} \approx \mathcal{O}\left(10^{-2}\right)$, and $\epsilon_{0}=8 \times 10^{-12}$. 

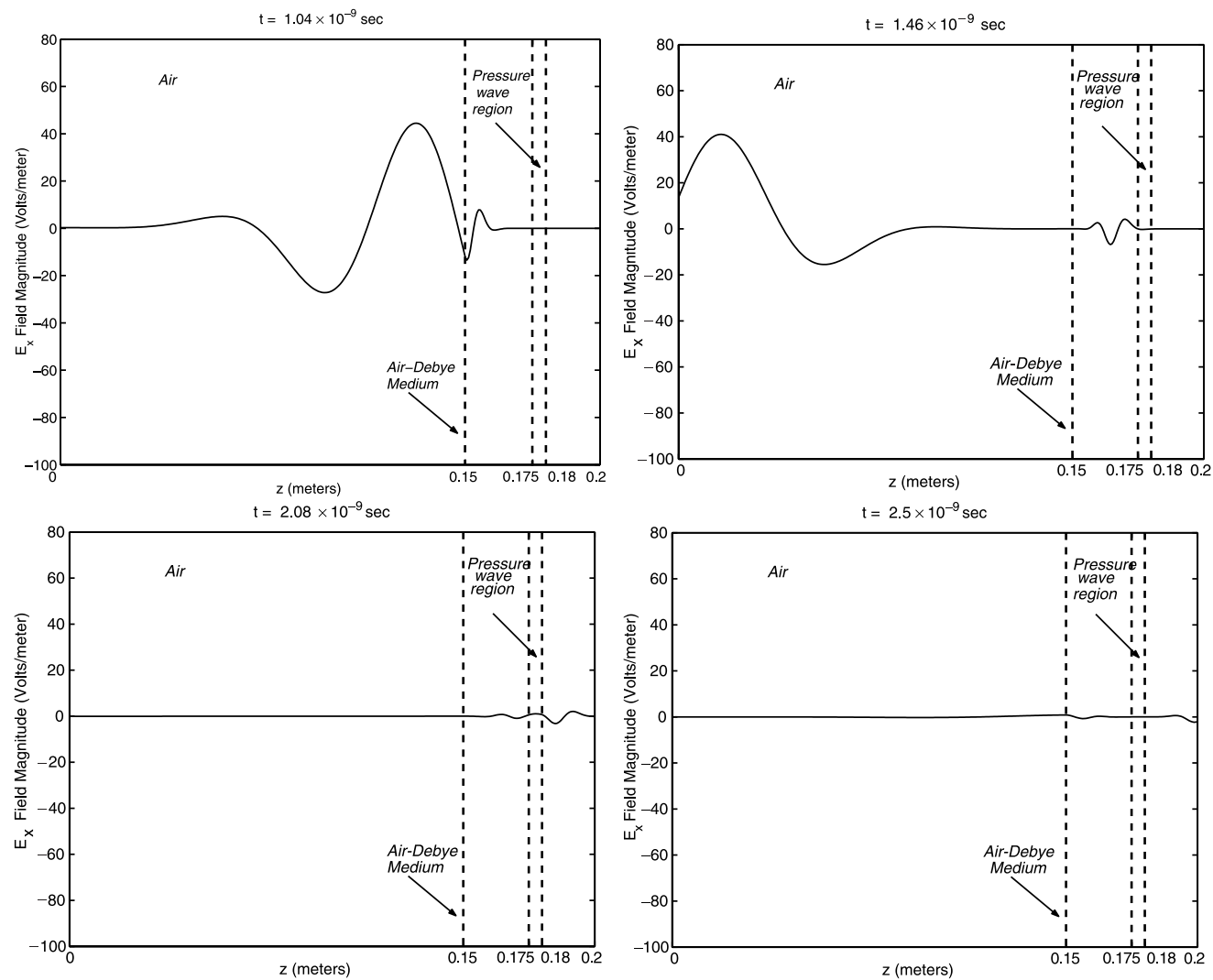

FIG. 6. Forward simulation for a Debye medium with parameters given in (47) and 48. Plot of the magnitude of the $E_{x}$ component of the electric field against $z$ (meters). (Top, left) The input signal gets partially reflected off the Debye medium and partially transmitted into the medium. (Top, right) The reflection off the air-Debye interface is seen moving towards the antenna, while the transmitted part of the source is seen propagating in the Debye medium towards the region containing the pressure wave. (Bottom, left) The interaction of the electromagnetic wave with the pressure wave causes the electromagnetic wave to partially reflect and partially transmit. (Bottom, right) The reflection from the acoustic pressure wave impinges on the air-Debye interface, while the wave transmitted into the pressure region moves into the right absorbing layer.

Rewriting (31) as

$$
\epsilon_{r}^{*}=\frac{\epsilon_{s}}{1+\mathrm{j} \omega \tau}+\left(\frac{\mathrm{j} \omega \tau}{1+\mathrm{j} \omega \tau}\right) \epsilon_{\infty}+\frac{\sigma}{\mathrm{j} \omega \epsilon_{0}}
$$

we consider each of the three terms in (51) separately to determine their magnitude. The magnitudes of the corresponding true values of these three terms (neglecting the acoustic 
pressure terms) are approximately given as

$$
\begin{array}{lll}
\left(\frac{\epsilon_{s, 0}^{*}}{1+\mathrm{j} \omega \tau_{0}^{*}}\right) & \approx \mathcal{O}\left(\epsilon_{s, 0}^{*}\right) & \approx \mathcal{O}(10), \\
\left(\frac{\mathrm{j} \omega \tau_{0}^{*}}{1+\mathrm{j} \omega \tau_{0}^{*}}\right) \epsilon_{\infty, 0}^{*} & \approx \mathcal{O}\left(10^{-2} \epsilon_{\infty, 0}^{*}\right) & \approx \mathcal{O}\left(10^{-2}\right), \\
\left(\frac{\sigma^{*}}{\mathrm{j} \omega \epsilon_{0}}\right) & \approx \mathcal{O}\left(10^{2} \sigma^{*}\right) & \approx \mathcal{O}\left(10^{-3}\right) .
\end{array}
$$

Thus we see that $\epsilon_{r}^{*}$ will be most sensitive to the static permittivity $\epsilon_{s, 0}$ and the effects of $\epsilon_{\infty, 0}$ and $\tau_{0}$ will not be as pronounced. Moreover, this implies that $\epsilon_{r}^{*}$ will be more sensitive to the pressure coefficient $\kappa_{s}$ than to the coefficients $\kappa_{\infty}$ and $\kappa_{\tau}$.

We are also interested in determining the effect that the acoustic speed and frequency have on the amplitude of the reflection from the acoustic pressure region. By changing the speed or the frequency, the wavelength $\lambda_{p}$ changes and thus the size of the interval $\left(z_{2}, z_{2}+\lambda_{p}\right)$ in which the pressure wave is generated. In [BB04b] we have shown that the amplitude of the acoustic reflection appears to decrease as the acoustic frequency is increased and increases at the acoustic speed increases. We note here that our windowed pressure wave contains only one wavelength of the sinusoid. Thus in general it will be difficult to predict precisely how the reflections will behave as the speed or the frequency is changed.

5. Parameter estimation and statistical inference: The inverse problem. In our forward simulations the signal that we record at the center of the antenna is a set of measurements of $E_{x}$, the $x$ component of the electric field, at the point $\left(x_{c}, z_{c}\right)$ in our computational domain at discrete, uniformly spaced intervals of time. This signal has segments consisting of the input source, the reflection at the air-Debye interface and another reflection from the region that contains the pressure wave as seen in Section 4 The reflected signal is a function of the various dielectric properties of the Debye medium; namely, the static relative permittivity $\epsilon_{s, 0}$, the infinite frequency permittivity $\epsilon_{\infty, 0}$, the relaxation time $\tau_{0}$, the conductivity $\sigma$, and the three pressure coefficients $\kappa_{s}$, $\kappa_{\infty}$ and $\kappa_{\tau}$. We collect all these quantities together to define the parameter vector

$$
\mathbf{q}=\left[\epsilon_{s, 0}, \epsilon_{\infty, 0}, \tau_{0}, \sigma, \kappa_{s}, \kappa_{\infty}, \kappa_{\tau}\right]^{T} .
$$

Thus, the signal recorded in the forward simulation is a function of $\mathbf{q}$. In general, such signals are usually obtained in an experimental setting conducted in a laboratory with physical equipment such as electric pulse generators to generate electric pulses, piezoelectric transducers that generate the acoustic pressure waves and antennas/receivers that record the electric field intensities. Moreover, such signals generated in an experimental setting are usually noisy, with noise arising through the equipment that is used; namely the resistor, antenna/receiver and the transducer, in our case. Implicit in modelling such a collection of measurements is the assumption that there exist true values of all the parameters that characterize the medium to be interrogated. We will denote the corresponding true parameter vector by $\mathbf{q}^{*}$. 
In order to simulate an experimental situation (i.e., generate typical "data"), we use these true values (which of course would not be known in an experimental setting!) in a forward simulation, and record the signal observed at the antenna as described above. We then add noise to the generated signal to produce a noisy signal which will form the observations or data. We use simulated data in the inverse problem for estimation of $\mathbf{q}^{*}$ to validate the methods first on "data" from known parameters in a setting with known noise. If we cannot estimate the dielectric parameters from data that is constructed via numerical simulations of our discrete model, then the reconstruction of these parameters from actual experimental data usually will not be feasible.

We can state the inverse or parameter estimation problem that we will attempt to solve as follows: Using observations or data (electric field intensities containing noise collected at the center of the antenna), determine an estimate $\hat{\mathbf{q}}$, of the true parameter $\mathbf{q}^{*}$, that belongs to an admissible set $Q$, so that the solution of the forward problem with $\mathbf{q}=\hat{\mathbf{q}}$ best describes the data that is collected. When we have solved this deterministic problem (as will be detailed below), it will also be necessary to specify reliability of our estimates, i.e., can we specify measures of uncertainty related to the estimate $\hat{\mathbf{q}}$ of $\mathbf{q}^{*}$ ? It is important to note that the uncertainty in consideration is inherent in the method of producing the estimates as well as in the process of data collection. Such measures of uncertainty will be specified by means of confidence intervals. These intervals are a probability statement about the inverse problem and the procedure which is used to construct estimates of the parameters. Thus we are led in a completely natural way to stochastic or probabilistic aspects of estimates from a deterministic problem solved with deterministic algorithms $[\mathrm{BB}$. The statistical error analysis that we will present here is based on standard statistical formulations as given in [DG95].

We first consider the two different ways in which we can add noise to our signal, i.e., the values of the $x$ component, $E_{x}$, of the electric field observed at the center of the antenna, $\left(x_{c}, z_{c}\right)$, for different times $t_{k}=k \Delta t, k=1, \ldots, M$. We represent this signal as the vector

$$
\mathbf{E}\left(\mathbf{q}^{*}\right)=\left\{E_{k}^{*}\right\}_{k=1}^{M}=\left(E_{x}\left(t_{1}, x_{c}, z_{c} ; \mathbf{q}^{*}\right), \ldots, E_{x}\left(t_{M}, x_{c}, z_{c} ; \mathbf{q}^{*}\right)\right)^{T},
$$

where $\mathbf{q}^{*}=\left(\epsilon_{s, 0}^{*}, \epsilon_{\infty, 0}^{*}, \tau_{0}^{*}, \sigma^{*}, \kappa_{s}^{*}, \kappa_{\infty}^{*}, \kappa_{\tau}^{*}\right)^{T}$ is the true parameter vector.

(1) Relative random noise $(R N)$ : In this case the amplitude of the noise that is added is proportional to the size of the signal, $\left\{E_{k}^{*}\right\}_{k=1}^{M}$. Our simulated data samples are

$$
O_{k}^{s}=E_{k}^{*}\left(1+\nu \eta_{k}^{s}\right), k=1, \ldots M,
$$

where $\bar{\eta}=\left\{\eta_{k}\right\}_{k=1}^{M}$ are independent normally distributed random variables with mean zero and variance one, i.e., $\eta_{k} \sim \mathcal{N}(0,1), k=1, \ldots, M$. We express the relative magnitude of the noise as a percentage of the magnitude of $\mathbf{E}\left(\mathbf{q}^{*}\right)$ by taking two times the value of $\nu$ as the size of the random variable. For example, $\nu=0.005$ corresponds to $1 \%$ relative noise [BBL00. This noise model does not produce constant variance across the samples.

(2) Constant variance noise: Since constant variance is most conveniently assumed in standard error analysis, we will consider estimates obtained from an inverse problem applied to simulated data which contain constant variance random noise. 
The data that we try to fit in this case is

$$
O_{k}^{s}=E_{k}^{*}+\beta \eta_{k}^{s}, k=1, \ldots M
$$

where as in (155), $\eta_{k} \sim \mathcal{N}(0,1), k=1, \ldots, M$. The constant $\beta$ is taken to be the product $\nu \alpha$, where $\alpha$ is a scaling factor which is chosen so that the signal to noise ratio (SNR) of the data defined in (56) corresponds to that of the data defined in (55) with noise level $\nu$. This justifies comparing results obtained with the two different ways of adding comparable noise to our signal. This will be addressed in more detail in Section 6.2.

The vector $\mathbf{O}^{s}=\left\{O_{k}^{s}\right\}_{k=1}^{M}$ will be our data or observations, and the noise $\bar{\eta}^{s}=\left\{\eta_{k}^{s}\right\}_{k=1}^{M}$ will be referred to as the measurement errors corresponding to the observations. The statistical error analysis that we now develop is only applicable to the case of constant variance noise. Thus, for the rest of this section, we will assume that our observations are of the form (56). The sample observations $\mathbf{O}^{s}$, which are in general obtained from experiment, are a realization of the corresponding random variable $\mathbf{O}=\left(O_{1}, O_{2}, \ldots, O_{M}\right)^{T}$ which can be seen to be a transformation of the random variable $\bar{\eta}$. Thus

$$
O_{k}=E_{k}^{*}+\beta \eta_{k}, k=1, \ldots M
$$

is a stochastic process and has a multivariate normal distribution with mean vector $\mathbf{E}\left(\mathbf{q}^{*}\right)$ defined in (54) and covariance matrix $\beta^{2} I_{M}$. The statistical model

$$
\mathbf{O} \sim \mathcal{N}_{M}\left\{\mathbf{E}\left(\mathbf{q}^{*}\right), \beta^{2} \mathcal{I}_{M}\right\}
$$

is a formal representation of the population of all possible realizations of $O_{1}, O_{2}, \ldots, O_{M}$ that can be observed. When we collect data, we are observing a single realization $O_{1}^{s}, \ldots, O_{M}^{s}$, i.e., a sample. Our objective then is to estimate the true value of the parameter $\mathbf{q}^{*}$ by collecting data, i.e., observing a single realization of $\mathbf{O}$ as well as accounting for the fact that a different realization will produce a different estimate of $\mathbf{q}^{*}$. We would like to learn about the true value $\mathbf{q}^{*}$ (which determines the nature of the population) from a sample, as well as indicate the certainty (or uncertainty) that we can associate to our knowledge of this parameter. This process of making statements about a population of interest on the basis of a sample from the population is called statistical inference.

The unknown true parameter vector $\mathbf{q}^{*}$ will be estimated by means of a suitable function $\hat{\mathbf{q}}(\mathbf{O})$ of the observations $\mathbf{O}$. The function $\hat{\mathbf{q}}(\mathbf{O})$ is a random variable and is referred to as an estimator. When evaluated at a particular realization, $O_{1}^{s}, O_{2}^{s}, \ldots, O_{M}^{s}$, this estimator yields a numerical value that gives information about the true value of the parameter $\mathbf{q}^{*}$. For a particular realization $\mathbf{O}^{s}$ of the random variable of observations $\mathbf{O}$, we call $\hat{\mathbf{q}}\left(\mathbf{O}^{s}\right)$ an estimate. In this paper we will consider the method of least squares to obtain estimates for the parameters and to calculate confidence intervals for the estimates by linearizing around the estimate $\hat{\mathbf{q}}\left(\mathbf{O}^{s}\right)$. In this case $\hat{\mathbf{q}}(\mathbf{O})$ will be the least squares estimator $\hat{\mathbf{q}}_{\mathrm{OLS}}(\mathbf{O})$. Associated with the random variable $\hat{\mathbf{q}}_{\mathrm{OLS}}(\mathbf{O})$ is the probability space $\mathcal{Q}=(Q, \mathcal{B}, m)$, where $Q$ is the sample set of all admissible parameters $\mathbf{q}, \mathcal{B}$ is the $\sigma$-algebra of events, and $m$ is the probability measure (or distribution). Thus we look 
for the least squares estimator $\hat{\mathbf{q}}_{\mathrm{OLS}}(\mathbf{O})$ on $Q$ such that

$$
\begin{aligned}
& \hat{\mathbf{q}} \mathrm{OLS}(\mathbf{O})=\arg \min _{\mathbf{q} \in Q} \mathrm{~J}(\mathbf{q}), \\
& \mathrm{J}(\mathbf{q})=\frac{1}{2} \sum_{k=1}^{M}\left|E_{x}\left(t_{k}, x_{c}, z_{c} ; \mathbf{q}\right)-O_{k}\right|^{2},
\end{aligned}
$$

where $\mathbf{E}(\mathbf{q})=\left(E_{x}\left(t_{1}, x_{c}, z_{c} ; \mathbf{q}\right), E_{x}\left(t_{2}, x_{c}, z_{c} ; \mathbf{q}\right), \ldots, E_{x}\left(t_{M}, x_{c}, z_{c} ; \mathbf{q}\right)\right)^{T}$ will be generated by our forward simulation. We will refer to the vector $\mathbf{E}(\mathbf{q})$ as our simulations. For a particular realization $\mathbf{O}^{s}$ of the random variable $\mathbf{O}$ of observations, this process will yield the least squares estimate $\hat{\mathbf{q}}_{\mathrm{OLS}}\left(\mathbf{O}^{s}\right) \in Q$, where

$$
\begin{aligned}
& \hat{\mathbf{q}}_{\mathrm{OLS}}\left(\mathbf{O}^{s}\right)=\arg \min _{\mathbf{q} \in Q} \mathrm{~J}^{S}(\mathbf{q}), \\
& \mathrm{J}^{S}(\mathbf{q})=\frac{1}{2} \sum_{k=1}^{M}\left|E_{x}\left(t_{k}, x_{c}, z_{c} ; \mathbf{q}\right)-O_{k}^{s}\right|^{2} .
\end{aligned}
$$

5.1. Calculation of confidence intervals via linearization. In general, the mappings between the parameters $\mathbf{q}$ and the simulations $\mathbf{E}$ are nonlinear. Let $E_{k}=E_{x}\left(t_{k}, x_{c}, z_{c} ; \mathbf{q}\right)$. The functions

$$
E_{k}\left(q_{1}, \ldots, q_{l}\right)=E_{x}\left(t_{k}, x_{c}, z_{c} ; \mathbf{q}\right), k=1, \ldots, M,
$$

denote real-valued differentiable functions of the unknown parameters $\mathbf{q}=\left(q_{1}, \ldots, q_{l}\right)^{T}$, where $1 \leq l \leq 7$ depending on how many parameters we attempt to identify. Let $\mathbf{q}=\mathbf{q}^{*}+\mathcal{D}_{\mathbf{q}}$, where the corrector term $\mathcal{D}_{\mathbf{q}}=\left(\Delta q_{1}, \ldots \Delta q_{l}\right)^{T}$ is unknown. Linearizing the functions in (61) around the true parameter $\mathbf{q}^{*}$ by using the Taylor expansion, we obtain

$$
\begin{aligned}
E_{k}\left(q_{1}, \ldots q_{l}\right) & =E_{k}\left(q_{1}^{*}+\Delta q_{1}, \ldots, q_{l}^{*}+\Delta q_{l}\right) \\
& =E_{k}\left(\mathbf{q}^{*}\right)+\left.\sum_{j=1}^{l} \frac{\partial E_{k}}{\partial q_{j}}\right|_{\mathbf{q}^{*}} \Delta q_{j}, k=1, \ldots, M .
\end{aligned}
$$

Let us define

$$
\mathcal{D}_{\mathrm{e}}=\left(O_{1}-E_{1}\left(\mathbf{q}^{*}\right), \ldots, O_{M}-E_{M}\left(\mathbf{q}^{*}\right)\right)^{T},
$$

and

$$
\mathcal{X}\left(\mathbf{q}^{*}\right)=\left(\begin{array}{ccc}
\left.\frac{\partial E_{1}}{\partial q_{1}}\right|_{\mathbf{q}^{*}} & \cdots & \left.\frac{\partial E_{1}}{\partial q_{l}}\right|_{\mathbf{q}^{*}} \\
\vdots & \vdots & \vdots \\
\left.\frac{\partial E_{M}}{\partial q_{1}}\right|_{\mathbf{q}^{*}} & \cdots & \left.\frac{\partial E_{M}}{\partial q_{l}}\right|_{\mathbf{q}^{*}}
\end{array}\right) .
$$

Thus, in order to estimate the unknown corrector terms by the method of least squares, the estimator $\hat{\mathcal{D}}_{\text {OLS }}$ is determined by

$$
\hat{\mathcal{D}}_{\mathrm{OLS}}=\min _{\mathcal{D}_{q}} \frac{1}{2}\left(\mathcal{D}_{\mathrm{e}}-\mathcal{X}\left(\mathbf{q}^{*}\right) \mathcal{D}_{\mathbf{q}}\right)^{T}\left(\mathcal{D}_{\mathrm{e}}-\mathcal{X}\left(\mathbf{q}^{*}\right) \mathcal{D}_{\mathbf{q}}\right),
$$

which is the linearized version of (59). This involves solving

$$
\frac{\partial\left(\mathcal{D}_{e}^{T} \mathcal{D}_{e}-2 \mathcal{D}_{e}^{T} \mathcal{X}\left(\mathbf{q}^{*}\right) \hat{\mathcal{D}}_{\mathrm{OLS}}+\hat{\mathcal{D}}_{\mathrm{OLS}}^{T} \mathcal{X}\left(\mathbf{q}^{*}\right)^{T} \mathcal{X}\left(\mathbf{q}^{*}\right) \hat{\mathcal{D}}_{\mathrm{OLS}}\right)}{\partial \hat{\mathcal{D}}_{\mathrm{OLS}}}=0
$$


and the corresponding optimality condition yields the estimator

$$
\hat{\mathcal{D}}_{\mathrm{OLS}}=\left(\mathcal{X}\left(\mathbf{q}^{*}\right)^{T} \mathcal{X}\left(\mathbf{q}^{*}\right)\right)^{-1} \mathcal{X}\left(\mathbf{q}^{*}\right)^{T} \mathcal{D}_{\mathrm{e}} .
$$

We note that the expected value of $\hat{\mathcal{D}}_{\text {OLS }}$ is $\operatorname{Exp}\left(\hat{\mathcal{D}}_{\text {OLS }}\right)=0$, and the covariance matrix of $\hat{\mathcal{D}}_{\text {OLS }}$ and hence also of $\hat{\mathbf{q}}_{\text {OLS }}$ is

$$
\begin{aligned}
\operatorname{Cov}\left(\hat{\mathcal{D}}_{\mathrm{OLS}}\right) & =\left(\mathcal{X}\left(\mathbf{q}^{*}\right)^{T} \mathcal{X}\left(\mathbf{q}^{*}\right)\right)^{-1} \mathcal{X}\left(\mathbf{q}^{*}\right)^{T} \operatorname{Cov}\left(\mathcal{D}_{\mathrm{e}}\right) \mathcal{X}\left(\mathbf{q}^{*}\right)\left(\mathcal{X}\left(\mathbf{q}^{*}\right)^{T} \mathcal{X}\left(\mathbf{q}^{*}\right)\right)^{-1} \\
& =\beta^{2}\left(\mathcal{X}\left(\mathbf{q}^{*}\right)^{T} \mathcal{X}\left(\mathbf{q}^{*}\right)\right)^{-1}=\operatorname{Cov}\left(\hat{\mathbf{q}}_{\mathrm{OLS}}\right),
\end{aligned}
$$

where $\hat{\mathbf{q}}_{\text {OLS }}=\mathbf{q}^{*}+\hat{\mathcal{D}}_{\text {OLS }}$, and $\beta$ is defined in (56). Thus the least squares estimator is a random variable with a multivariate normal distribution which after linearization can be approximately represented as

$$
\hat{\mathbf{q}}_{\mathrm{OLS}}(\mathbf{O}) \sim \mathcal{N}_{l}\left(\mathbf{q}^{*}, \beta^{2}\left[\mathcal{X}^{T}\left(\mathbf{q}^{*}\right) \mathcal{X}\left(\mathbf{q}^{*}\right)\right]^{-1}\right) .
$$

However, when data is generated in an experimental setting, we do not have any knowledge of the true parameter vector $\mathbf{q}^{*}$ and hence we cannot calculate $\left(\mathcal{X}\left(\mathbf{q}^{*}\right)^{T} \mathcal{X}\left(\mathbf{q}^{*}\right)\right)^{-1}$. Thus, we further approximate our least squares estimator as having the multivariate normal distribution

$$
\hat{\mathbf{q}}_{\mathrm{OLS}}(\mathbf{O}) \sim \mathcal{N}_{l}\left(\hat{\mathbf{q}}_{\mathrm{OLS}}\left(\mathbf{O}^{s}\right), \hat{\beta}_{\mathrm{OLS}}^{2}\left[\mathcal{X}^{T}\left(\hat{\mathbf{q}}_{\mathrm{OLS}}\left(\mathbf{O}^{s}\right)\right) \mathcal{X}\left(\hat{\mathbf{q}}_{\mathrm{OLS}}\left(\mathbf{O}^{s}\right)\right)\right]^{-1}\right),
$$

which is obtained by repeating the linearization process around the estimate $\hat{\mathbf{q}}_{\mathrm{OLS}}\left(\mathbf{O}^{s}\right)$ obtained from a realization $\mathbf{O}^{s}$ of the random variable $\mathbf{O}$ of observations. The value of $\hat{\beta}_{\text {OLS }}^{2}$ is chosen as

$$
\hat{\beta}_{\mathrm{OLS}}^{2}=\frac{1}{M-l} \sum_{k=1}^{M}\left|E_{x}\left(t_{k}, x_{c}, z_{c} ; \hat{\mathbf{q}} \mathrm{OLS}\left(\mathbf{O}^{s}\right)\right)-O_{k}^{s}\right|^{2},
$$

which is the minimum value of the least squares objective function scaled by $2 /(M-l)$.

Whenever an estimate based on data is reported, it should be accompanied by an assessment of uncertainty based on the sampling distribution. To this end we will construct confidence intervals for all the estimates that we will provide. The approach we use is to look at the standard error (SE) for each of the $l$ components of the estimate $\hat{\mathbf{q}}_{\mathrm{OLS}}\left(\mathbf{O}^{s}\right)$, which is given for its $j$ th component by the $j$ th diagonal term of the covariance matrix, i.e.,

$$
\begin{aligned}
\operatorname{SE}\left(\hat{q}_{\mathrm{OLS}, j}\right) & =\sqrt{\operatorname{var}\left(\hat{q}_{\mathrm{OLS}, j}\left(\mathbf{O}^{s}\right)\right)} \\
& =\sqrt{\hat{\beta}_{\mathrm{OLS}}^{2}\left(\left(\mathcal{X}\left(\hat{\mathbf{q}}_{\mathrm{OLS}}\left(\mathbf{O}^{s}\right)\right)^{T} \mathcal{X}\left(\hat{\mathbf{q}}_{\mathrm{OLS}}\left(\mathbf{O}^{s}\right)\right)\right)^{-1}\right)_{j j}} j=1, \ldots, l .
\end{aligned}
$$

We construct the intervals

$$
\mathrm{CI}_{j}=\left(\hat{q}_{\mathrm{OLS}, j}\left(\mathbf{O}^{s}\right)-1.96 S E\left(\hat{q}_{\mathrm{OLS}, j}\right), \quad \hat{q}_{\mathrm{OLS}, j}\left(\mathbf{O}^{s}\right)+1.96 S E\left(\hat{q}_{\mathrm{OLS}, j}\right)\right), j=1, \ldots, l,
$$

for which

$$
\begin{aligned}
& m\left(\left\{\hat{q}_{\mathrm{OLS}, j}\left(\mathbf{O}^{s}\right)-1.96 S E\left(\hat{q}_{\mathrm{OLS}, j}\right)<\hat{q}_{\mathrm{OLS}, j}(\mathbf{O})<\hat{q}_{\mathrm{OLS}, j}\left(\mathbf{O}^{s}\right)+1.96 S E\left(\hat{q}_{\mathrm{OLS}, j}\right)\right\}\right)=0.95, \\
& \quad j=1, \ldots, l,
\end{aligned}
$$


where $m$ is the probability measure for the probability space $\mathcal{Q}$ and $M$ is sufficiently large that one can use a Gaussian $\mathcal{N}(0,1)$ distribution in computing confidence intervals. These intervals $\mathrm{CI}_{j}$ are called confidence intervals with confidence level 0.95 or the $95 \%$ confidence interval.

REMARK 2. The confidence intervals are a probability statement about the procedure by which an estimate is constructed from a sample of the population. They are not a probability statement about the true parameter $\mathbf{q}^{*}$ which is fixed. If we could construct the confidence intervals, from our estimation procedure, for all possible data samples of size $\mathrm{M}$, then $95 \%$ of such intervals would cover the true parameter values $\mathbf{q}^{*}$. However, for a particular data sample $\mathbf{O}^{s}$, the confidence intervals constructed as above may or may not cover the true parameter $\mathbf{q}^{*}$. What we can state is that we are $95 \%$ confident that the confidence intervals constructed by our estimation procedure would cover $\mathbf{q}^{*}$.

6. Parameter estimation: First test problem. We now attempt to estimate the dielectric parameters for the Debye medium defined by (47) and (48). In particular, the values of all the parameters are

$$
\begin{aligned}
\epsilon_{s, 0}^{*} & =80.1 & \kappa_{s}^{*} & =48.06 \\
\epsilon_{\infty, 0}^{*} & =5.5 & \kappa_{\infty}^{*} & =4.4 \\
\tau_{0}^{*} & =8.1 \times 10^{-12} & \kappa_{\tau}^{*} & =4.05 \times 10^{-13} . \\
\sigma^{*} & =1 \times 10^{-5} & &
\end{aligned}
$$

We will refer to the values (75) as the true values of the parameters. We solved the corresponding least squares optimization problem using two different methods; the NelderMead algorithm, which is a simplex based, gradient free method, and the LevenbergMarquardt trust region method that uses forward finite differences to calculate the gradient. From our analysis in Section 4.1, we found that our problem is sensitive to only three of the seven parameters. We will then try to estimate two or three of the parameters to which our problem is sensitive. The particular implementation of the LevenbergMarquardt and the Nelder-Mead algorithm that are used for the calculations presented in this paper are based on the corresponding algorithms given in [Kel99.

6.1. Simulation results: The Nelder-Mead method. We first present parameter estimation results using the Nelder-Mead simplex based algorithm which is used for optimization in noisy problems. The Nelder-Mead simplex algorithm keeps a simplex $\mathcal{S}$ of approximations to an optimal point. In this algorithm the vertices of the simplex $\left\{\mathbf{q}_{i}\right\}_{i=1}^{l+1}$, where $l$ is the size of $\mathbf{q}$, are sorted according to the least squares objective function values

$$
\mathrm{J}^{S}\left(\mathbf{q}_{1}\right) \leq \mathrm{J}^{S}\left(\mathbf{q}_{2}\right) \leq \ldots \leq \mathrm{J}^{S}\left(\mathbf{q}_{l+1}\right) .
$$

The point $\mathbf{q}_{1}$ is called the best vertex and $\mathbf{q}_{l+1}$ the worst. The algorithm attempts to replace the worst vertex $\mathbf{q}_{l+1}$ with a new point of the form

$$
\mathbf{q}\left(\nu_{n m}\right)=\left(1+\nu_{n m}\right) \overline{\mathbf{q}}-\nu_{n m} \mathbf{q}_{l+1},
$$


TABLE 1. Final estimates for all seven parameters for water using Nelder-Mead. The initial parameter simplex has values that are 5$10 \%$ larger than the true values.

\begin{tabular}{|c|c|c|}
\hline Parameter & True Values & Final Estimates \\
\hline$\epsilon_{s, 0}$ & 80.1 & 80.1111 \\
$\epsilon_{\infty, 0}$ & 5.5 & 5.7461 \\
$\tau_{0}$ & $8.1 \times 10^{-12}$ & $8.1283 \times 10^{-12}$ \\
$\sigma$ & $1.0 \times 10^{-5}$ & $1.1972 \times 10^{-5}$ \\
$\kappa_{s}$ & 48.06 & 48.1227 \\
$\kappa_{\infty}$ & 4.4 & 4.9148 \\
$\kappa_{\tau}$ & $4.05 \times 10^{-13}$ & $4.1935 \times 10^{-13}$ \\
\hline
\end{tabular}

where $\overline{\mathbf{q}}$ is the centroid of the convex hull of $\left\{\mathbf{q}_{i}\right\}_{i=1}^{l}$

$$
\overline{\mathbf{q}}=\frac{1}{l} \sum_{i=1}^{l} \mathbf{q}_{i}
$$

The value of $\nu_{n m}$ is selected from a sequence of values that are computed by performing several different operations on the simplex. In general, the Nelder-Mead algorithm is not guaranteed to converge, even for smooth problems. The failure mode is stagnation at a nonoptimal point. For further details we refer the reader to [Kel99].

In the first test we will try to estimate all of seven parameters in $\mathbf{q}^{*}$. The initial simplex is chosen to have values that are 5-10\% larger than the true parameter values given in (75). We do not add any noise to our data in this first test. The final estimates from the Nelder-Mead algorithm are presented in Table 1, The Nelder-Mead algorithm is terminated when the maximum least squares function value difference between any two points in the simplex is less than $10^{-8}$. The algorithm converges in 283 iterations (for details, see $\mathrm{BB} 04 \mathrm{~b}]$ ).

The parameters $\epsilon_{\infty, 0}, \sigma$ and the two pressure coefficients $\kappa_{\infty}$ of $\epsilon_{\infty}$ and $\kappa_{\tau}$ of $\tau$ are difficult for the inverse problem to identify. On the other hand, we observed that the parameters $\epsilon_{s, 0}$ and its pressure coefficient $\kappa_{s}$ as well as the parameter $\tau_{0}$ are relatively well estimated. Since the inverse problem permits ready identification of only three parameters in the absence of noise, we do not expect to see any improvement when noise is added to the data! Thus we will henceforth concentrate on the identification of $\epsilon_{s, 0}$, $\tau_{0}$ and $\kappa_{s}$.

We will first attempt the identification of $\epsilon_{s, 0}$ and $\kappa_{s}$. In a first test, we fix all the other 5 parameters (including $\tau_{0}$ ) at the true values and attempt to identify $\epsilon_{s, 0}$ and $\kappa_{s}$. Table 2 presents the results for this test using several different initial simplices. As can be seen from Table 2, with the other 5 parameters fixed at their true values, we do not have any difficulty in identifying $\epsilon_{s, 0}$ and $\kappa_{s}$ accurately. In Table 2, FC denotes the function count, i.e., the number of times the least squares objective function is evaluated.

The algorithm converges when the difference between function values is less than $10^{-8}$. For the second case, where the initial simplex has points with 5\%-10\% larger values than 
TABle 2. Parameter estimation of $\epsilon_{s, 0}$ and $\kappa_{s}$ for different initial simplices with no added noise.

\begin{tabular}{|c|c|c|c|c|c|c|c|}
\hline $\mathbf{q}_{0}$ & Iter & $\hat{\epsilon}_{s, 0}$ & $\hat{\kappa}_{s}$ & $\left|J_{n+1}^{S}-\mathrm{J}_{0}^{S}\right|$ & $\mathrm{J}^{S}$ & $\left\|\nabla \mathrm{J}^{S}\right\|_{L^{2}}$ & $\mathrm{FC}$ \\
\hline $0.95 \mathbf{q}^{*}$ & 33 & 80.0999 & 48.0607 & $4.7019 \times 10^{-9}$ & $1.4560 \times 10^{-8}$ & 0.0204 & 65 \\
$1.05 \mathbf{q}^{*}$ & 32 & 80.1 & 48.06 & $9.5079 \times 10^{-9}$ & $7.1205 \times 10^{-27}$ & 0.0096 & 66 \\
$0.5 \mathbf{q}^{*}$ & 59 & 80.1001 & 48.0603 & $8.4746 \times 10^{-9}$ & $5.5171 \times 10^{-9}$ & 0.0130 & 112 \\
\hline
\end{tabular}

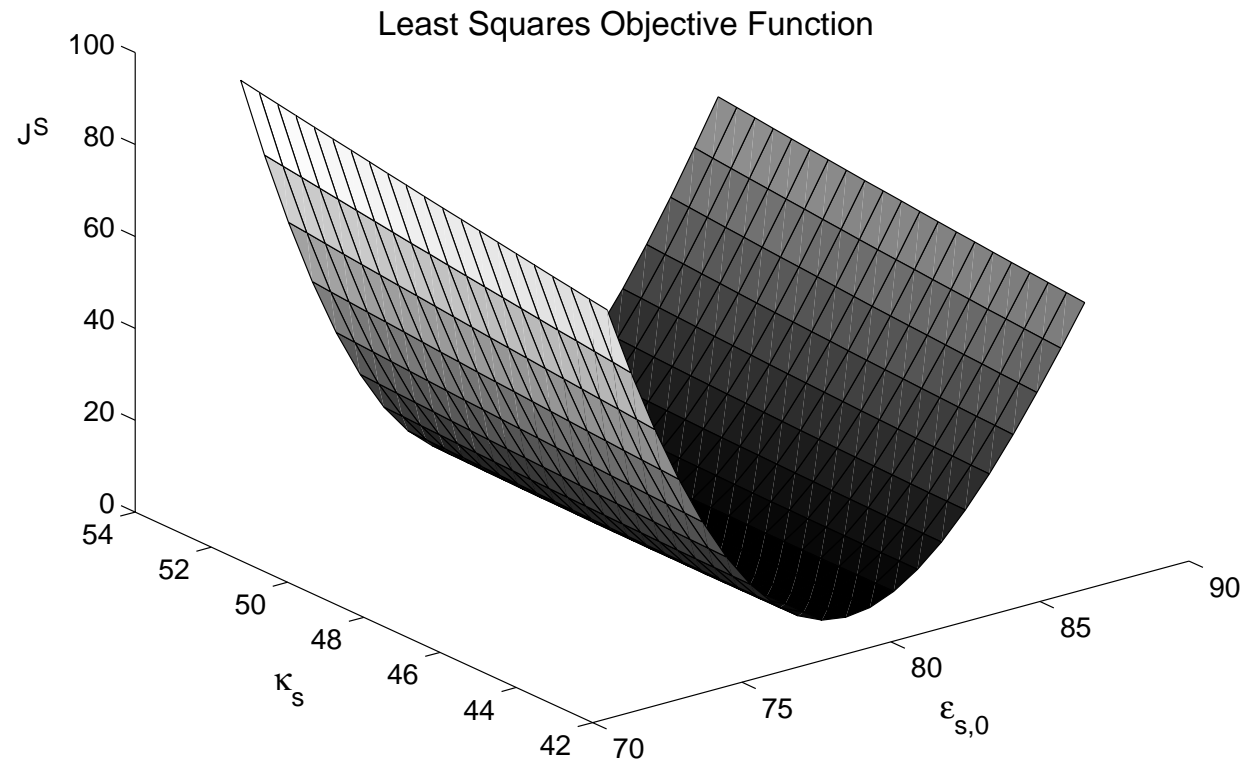

Fig. 7. The least squares objective function for different values of $\epsilon_{s, 0}$ and $\kappa_{s}$.

the true values, we obtain an unusually small least squares function value of the order of $10^{-27}$. We believe this to be an accidental artifact of the algorithm.

In Figures 7 and 8 we plot the least squares objective function for values of $\epsilon_{s, 0}$ and $\kappa_{s}$ that differ by $0 \%-10 \%$ from the true values. The other five parameters are kept fixed at their true values. From Figures 7 and 8 , we determine that our inverse problem is more sensitive to $\epsilon_{s, 0}$ than to the pressure coefficient $\kappa_{s}$. This is expected as the static relative permittivity, $\epsilon_{s, 0}$, is more influential in the system dynamics than the pressure coefficients, and hence is also more important in identifying and characterizing the material.

As explained in Section 5 in a more realistic situation we will not have access to the true values of any of the parameters. Hence, we now fix five of the seven parameters at values that have a relative error from the true values of about $10 \%-50 \%$. We use the 
Least Squares Objective Function Values
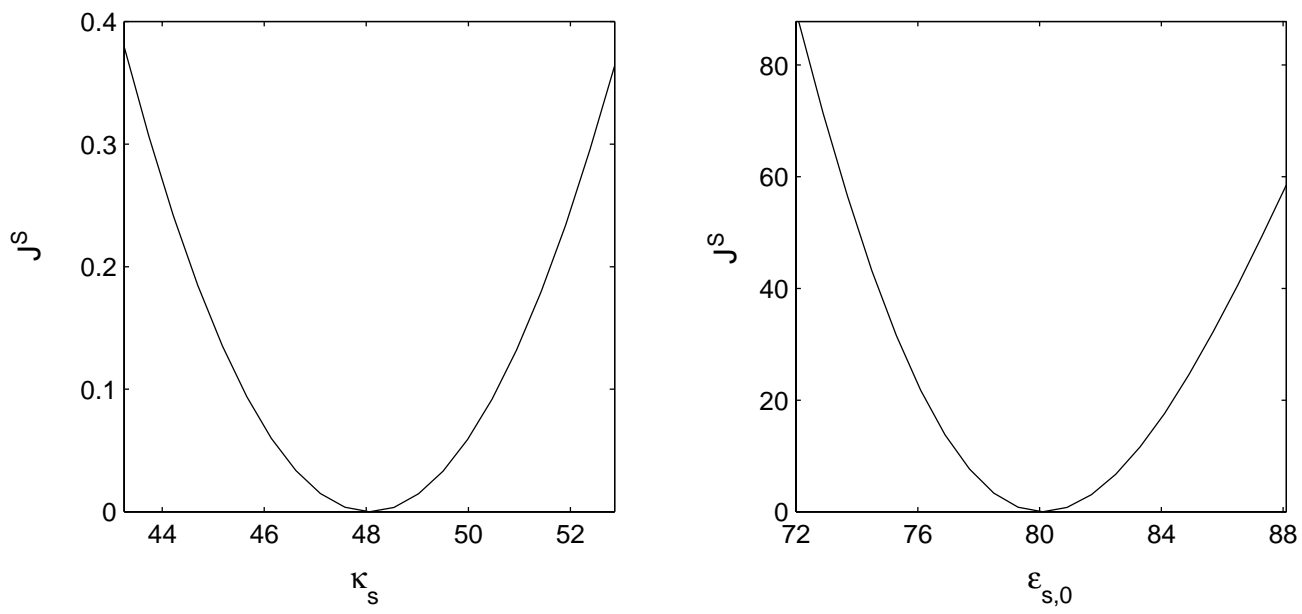

Fig. 8. Cross-sections from Figure 7 of the least squares objective function versus $\kappa_{s}$ (left) and versus $\epsilon_{s, 0}$ (right). Note the difference in scales between the two figures.

values

$$
\begin{array}{ll}
\epsilon_{\infty, 0} & =6.0, \\
\tau_{0} & =10.0 \times 10^{-12}, \\
\sigma & =1.5 \times 10^{-5}, \\
\kappa_{\infty} & =4.8 \\
\kappa_{\tau} & =5.0 \times 10^{-13}
\end{array}
$$

in our simulations. Such choices for the parameters are typical in testing algorithms BK89. We use different initial guesses for $\epsilon_{s, 0}$ and $\kappa_{s}$. As discussed earlier, noise is introduced into the data. We first add relative random noise to our data and we use the fixed values (79) in the inverse problem. The results are tabulated in Table 3

From Table 3 we see that using the inverse problem we are able to estimate $\epsilon_{s, 0}$ but we are unable to reconstruct $\kappa_{s}$ with good accuracy. Table 3 shows the final estimates of $\epsilon_{s, 0}$ and $\kappa_{s}$ for water. The other five parameters are fixed at the values given in (79) and relative noise of varying levels is added to the data.

To understand why $\kappa_{s}$ is not recovered, we note that the fixed value of $\tau_{0}$ in (79) has a relative error of $23 \%$ from its true value. Since the reflections from the acoustic

TABLE 3. Parameter estimation of $\epsilon_{s, 0}$ and $\kappa_{s}$ for $0 \%, 1 \%, 3 \%$ and $5 \%$ relative noise.

\begin{tabular}{|c|c|c|c|c|c|c|c|}
\hline$\% \mathrm{RN}$ & Iter & $\hat{\epsilon}_{s, 0}$ & $\hat{\kappa}_{s}$ & $\left|\mathrm{~J}_{n+1}^{S}-\mathrm{J}_{0}^{S}\right|$ & $\mathrm{J}^{S}$ & $\left\|\nabla \mathrm{J}^{S}\right\|_{L^{2}}$ & $\mathrm{FC}$ \\
\hline 0.0 & 37 & 80.3636 & 66.0486 & $8.5719 \times 10^{-9}$ & 2.5594 & 0.0076 & 73 \\
1.0 & 41 & 80.3638 & 66.0422 & $3.2939 \times 10^{-9}$ & 25.2195 & 0.0098 & 80 \\
3.0 & 40 & 80.3640 & 66.0280 & $8.6023 \times 10^{-9}$ & 208.1146 & 0.0080 & 77 \\
5.0 & 40 & 80.3643 & 66.0157 & $6.3563 \times 10^{-9}$ & 574.4429 & 0.0060 & 79 \\
\hline
\end{tabular}


TABLE 4. Parameter estimation of $\epsilon_{s, 0}$ and $\kappa_{s}$ for $0 \%-10 \%$ added relative random noise. The values of the other five parameters are fixed at values given in (80).

\begin{tabular}{|c|c|c|c|c|c|c|c|}
\hline$\%$ RN & Iter & $\hat{\epsilon}_{s, 0}$ & $\hat{\kappa}_{s}$ & $\left|\mathrm{~J}_{n+1}^{S}-\mathrm{J}_{0}^{S}\right|$ & $\mathrm{J}^{S}$ & $\left\|\nabla \mathrm{J}^{S}\right\|_{L^{2}}$ & $\mathrm{FC}$ \\
\hline 0.0 & 38 & 80.2165 & 55.2945 & $1.5487 \times 10^{-9}$ & 0.4369 & 0.0013 & 73 \\
1.0 & 39 & 80.2169 & 55.2914 & $4.5901 \times 10^{-9}$ & 23.2556 & 0.0075 & 76 \\
5.0 & 40 & 80.2178 & 55.2729 & $8.2103 \times 10^{-9}$ & 573.1135 & 0.0079 & 78 \\
10.0 & 39 & 80.2191 & 55.2509 & $2.5361 \times 10^{-9}$ & 2292.24730 & 0.00701 & 76 \\
\hline
\end{tabular}

region depend strongly on the value of $\tau_{0}$, a relative error of $23 \%$ is simply too high to get a good estimate of the pressure coefficients. To test this we lower the value of $\tau_{0}$ to $8.91 \times 10^{-12}$. So instead of the values in (79) we will use the values

$$
\begin{array}{ll}
\epsilon_{\infty, 0} & =6.0, \\
\tau_{0} & =8.91 \times 10^{-12} \\
\sigma & =1.5 \times 10^{-5} \\
\kappa_{\infty} & =4.8 \\
\kappa_{\tau} & =5.0 \times 10^{-13} .
\end{array}
$$

This value of $\tau_{0}$ has a relative error of $10 \%$ from its true value $\tau_{0}^{*}$. With this new fixed value for $\tau_{0}$ we attempt the inverse problem again to reconstruct $\hat{\epsilon}_{s, 0}$ and $\hat{\kappa}_{s}$. These results are presented in Table 4

From Table 4 we observe that the estimated value of $\kappa_{s}$ is closer to the true value than in the previous test. Thus we can obtain accurate estimates of $\kappa_{s}$ by choosing values of $\tau_{0}$ that are closer to its true value. We can conclude that if the value of $\tau_{0}$ is not accurately known, then the identification of $\kappa_{s}$ is difficult. The identification of $\epsilon_{s, 0}$, on the other hand, does not appear to be sensitive to the fixed value of $\tau_{0}$ that is chosen. The combined identification of both $\epsilon_{s, 0}$ and $\kappa_{s}$ appears to be quite insensitive to the level of relative noise that is added to the data.

We next attempt the inverse problem with simulated data to which we add constant variance noise. We would like to compare the results of this case with the results obtained from the inverse problem with relative random noise in the simulated data. We use the notion of signal to noise ratio to make such comparisons.

6.2. Signal to noise ratio. In analog and digital communications, the signal to noise ratio, denoted as SNR, is a measure of the signal strength (usually given in decibels (dB)) relative to the background noise. There are many equivalent ways of defining the SNR [JN84]. Since we assume the mean of our noise to be zero, we define the SNR as

$$
\mathrm{SNR}=10 \log _{10}\left(\frac{\sum_{k=1}^{M}\left|E_{k}^{*}\right|^{2}}{\sum_{k=1}^{M} \operatorname{var}\left(O_{k}-E_{k}^{*}\right)}\right) \mathrm{dB},
$$

where $\mathrm{dB}$ denotes decibels. If the signal strength is equal to the variance of the background noise, i.e.,

$$
\sum_{k=1}^{M}\left|E_{k}^{*}\right|^{2}=\sum_{k=1}^{M} \operatorname{var}\left(O_{k}-E_{k}^{*}\right)
$$


TABLE 5. Comparison of relative random noise and constant variance noise

\begin{tabular}{|c|l|l|c|}
\hline SNR $(\mathrm{dB})$ & $\nu$ & $\% \mathrm{RN}$ & $\beta=\nu \alpha$ \\
\hline 66 & 0.0005 & 0.1 & 0.0118 \\
46 & 0.005 & 1.0 & 0.1175 \\
32 & 0.025 & 5.0 & 0.5877 \\
26 & 0.05 & 10.0 & 1.1754 \\
\hline
\end{tabular}

then $\mathrm{SNR}=0$, and the signal is almost unreadable, as the noise level severely competes with it. Ideally, the strength of the signal is greater than the variance of the noise, so that the SNR is positive. The SNR ratio can sometimes be increased by providing the source with a higher level of signal output power if necessary. In wireless systems, it is always important to optimize the performance of the transmitting and receiving antennas.

Using the notation presented in Section 5 the SNR in the cases of relative random noise $\mathrm{SNR}_{\mathrm{R}}$ and constant variance noise $\mathrm{SNR}_{\mathrm{C}}$ are, respectively, given as

$$
\begin{aligned}
& \mathrm{SNR}_{\mathrm{R}}=10 \log _{10}\left(\frac{\sum_{k=1}^{M}\left|E_{k}^{*}\right|^{2}}{\sum_{k=1}^{M} \operatorname{var}\left(\nu E_{k}^{*} \eta_{k}\right)}\right) \mathrm{dB}, \\
& \mathrm{SNR}_{\mathrm{C}}=10 \log _{10}\left(\frac{\sum_{k=1}^{M}\left|E_{k}^{*}\right|^{2}}{\sum_{k=1}^{M} \operatorname{var}\left(\beta \eta_{k}\right)}\right) \mathrm{dB} .
\end{aligned}
$$

To compare the case of constant variance noise with the case of relative random noise, we proceed as follows.

(1) As noted in (56) and the discussion thereafter, we set $\beta=\nu \alpha$. Setting the SNR in the two cases to be equal, i.e., $\mathrm{SNR}_{\mathrm{R}}=\mathrm{SNR}_{\mathrm{C}}$, implies

$$
\sum_{k=1}^{M} \operatorname{var}\left(\nu E_{k}^{*} \eta_{k}\right)=M \nu^{2} \alpha^{2} \Longrightarrow \alpha=\sqrt{\frac{\sum_{k=1}^{M}\left|E_{k}^{*}\right|^{2}}{M}} .
$$

(2) Thus for a given value of $\nu$, we have $\beta=\alpha \nu$ and the corresponding SNR is calculated as

$$
\mathrm{SNR}_{\mathrm{R}} \equiv \mathrm{SNR}_{\mathrm{C}}=10 \log _{10}\left(\frac{\sum_{k=1}^{M}\left|E_{k}^{*}\right|^{2}}{M \alpha^{2} \nu^{2}}\right)=10 \log _{10}\left(\frac{1}{\nu^{2}}\right)
$$

Hence, for each value of $\nu$, there is a corresponding value of $\beta$ for which the SNR for relative random noise is equivalent to the SNR for constant variance noise. In Table 5 we present the values of the SNR, $\beta$, and $\nu$ that correspond to each other in the manner described above. For this test problem $\alpha=23.5077$.

We next attempt to identify the two parameters $\epsilon_{s, 0}$ and $\kappa_{s}$ by adding constant variance noise as given in (56) to our data and using the fixed values (801). The results for the corresponding inverse problem are presented in Table 6. The results in this case are quite different from the case of relative noise as shown in Table 4 We can see that the estimation of $\kappa_{s}$ becomes worse as the level of noise increases. The estimation of $\epsilon_{s, 0}$ on the other hand is relatively stable, though it is not as accurate as the estimate obtained in the case where relative noise is added to the data. The estimates of $\epsilon_{s, 0}$ also become 
worse as the level of constant variance noise increases. In Table 6 , $\nu$ corresponds to the scaling factor in (55). The constant variance case and the relative noise case for the same value of $\nu$ have the same signal to noise ratio.

We now calculate confidence intervals for the estimates that we obtained in Table 6 The procedure for calculating these intervals was outlined in Section 5.1. These intervals are presented in Table 7 for varying levels of noise. From our earlier discussion about confidence intervals, we note that the size of the intervals is in direct proportion to the level of uncertainty that we have about the estimates that we have obtained. As we can see from Table 7, we have tighter intervals for the estimates of $\epsilon_{s, 0}$ than we do for the estimates of $\kappa_{s}$, which is expected as our problem is more sensitive to $\epsilon_{s, 0}$ than it is to the pressure coefficient $\kappa_{s}$. We also notice that the intervals become larger as the level of noise that is added to the data is increased. These intervals correspond to a $95 \%$ confidence level.

Plots of the least squares objective function vs. $\left(\epsilon_{s, 0}, \kappa_{s}\right)$, with the other parameters fixed at either the true values or those in (80), and 1\% RN (constant variance) in the data, reveal that indeed the minimum value for $\kappa_{s}$ is no longer near the true value (see BB04b] for details).

TABLE 6. Parameter estimation of $\epsilon_{s, 0}$ and $\kappa_{s}$ for $0 \%-10 \%$ constant variance noise. The other five parameters are fixed at the values given in 80 ,

\begin{tabular}{|c|c|c|c|c|c|l|l|}
\hline \% RN & Iter & $\hat{\epsilon}_{s, 0}$ & $\hat{\kappa}_{s}$ & $\left|\mathrm{~J}_{n+1}^{S}-\mathrm{J}_{0}^{S}\right|$ & $\mathrm{J}^{S}$ & $\left\|\nabla \mathrm{J}^{S}\right\|_{L^{2}}$ & $\mathrm{FC}$ \\
\hline 0.0 & 38 & 80.2165 & 55.2945 & $1.5487 \times 10^{-9}$ & 0.4369 & 0.0013 & 73 \\
1.0 & 41 & 80.1654 & 56.4531 & $3.1244 \times 10^{-9}$ & 27.0778 & 0.007561 & 82 \\
5.0 & 39 & 79.9621 & 61.1779 & $7.8005 \times 10^{-9}$ & 667.7927 & 0.0111 & 79 \\
10.0 & 44 & 79.7084 & 67.3235 & $3.5288 \times 10^{-10}$ & 2670.5115 & 0.007417 & 85 \\
\hline
\end{tabular}

TABLE 7. Confidence intervals for the parameter estimation of $\epsilon_{s, 0}$ and $\kappa_{s}$ for constant variance noise with the Nelder-Mead algorithm.

\begin{tabular}{|c|c|}
\hline & $\mathrm{RN}=0.0 \%$ \\
\hline$\hat{\epsilon}_{s, 0}$ & $\left(8.02165 \pm 1.8804 \times 10^{-3}\right) \times 10$ \\
$\hat{\kappa}_{s}$ & $\left(5.52945 \pm 1.6448 \times 10^{-2}\right) \times 10$ \\
\hline & $\mathrm{RN}=1.0 \%$ \\
\hline$\hat{\epsilon}_{s, 0}$ & $\left(8.01654 \pm 1.4577 \times 10^{-2}\right) \times 10$ \\
$\hat{\kappa}_{s}$ & $\left(5.64531 \pm 1.2738 \times 10^{-1}\right) \times 10$ \\
\hline & $\mathrm{RN}=5.0 \%$ \\
\hline$\hat{\epsilon}_{s, 0}$ & $\left(7.99621 \pm 6.8206 \times 10^{-2}\right) \times 10$ \\
$\hat{\kappa}_{s}$ & $\left(6.11779 \pm 5.9447 \times 10^{-1}\right) \times 10$ \\
\hline & $\mathrm{RN}=10.0 \%$ \\
\hline$\hat{\epsilon}_{s, 0}$ & $\left(7.97084 \pm 1.2723 \times 10^{-1}\right) \times 10$ \\
$\hat{\kappa}_{s}$ & $(6.73235 \pm 1.1076) \times 10$ \\
\hline
\end{tabular}




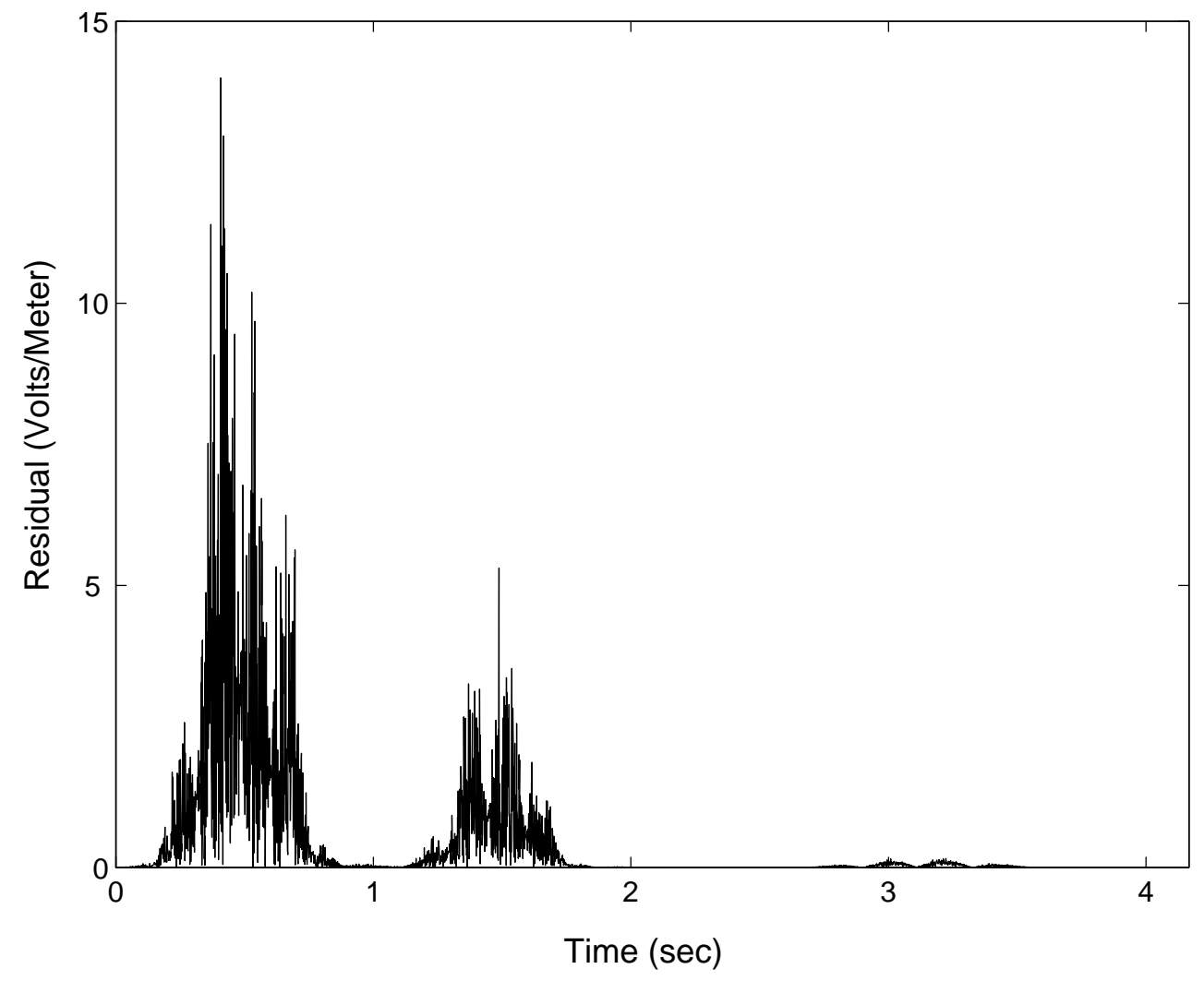

Fig. 9. Plot of the least squares residual versus time in the case of $10 \%$ relative random noise added to the data.

In Figure 9 we plot the least squares residual versus time in the case when $10 \%$ relative random noise is added to the data. A magnified plot of the least squares residual versus time in the interval where the acoustic reflection is observed is plotted in Figure 10 .

Finally we plot the least squares residual versus the absolute value of the electric field data in Figure 11 again in the case where 10\% relative random noise is added to the data. We notice in this figure that the amount of noise increases as the absolute value of the electric field data magnitude increases. This is in direct contrast to the case when constant variance noise is added to the simulated data. We plot analogous plots of the least squares residual for $10 \%$ added constant variance noise in Figures 12 and 13 Comparing Figures 12 and 9, we note the lack of any patterns in the residual in Figure 12 for the case of added constant variance noise, whereas in Figure 9, the residual follows the peaks in the simulated data used in the inverse problem. Again in Figure 111 we note the fan-like structure of the residual with the residual increasing with the absolute value of the electric field. In the case of added constant variance noise, however, we again note the lack of a pattern in the residual as seen in Figure 13. We also observe that there are many points of the residual on the line $E_{x}=0$. This is because in the simulated data, the electric field magnitude is almost zero most of the time. We compare 


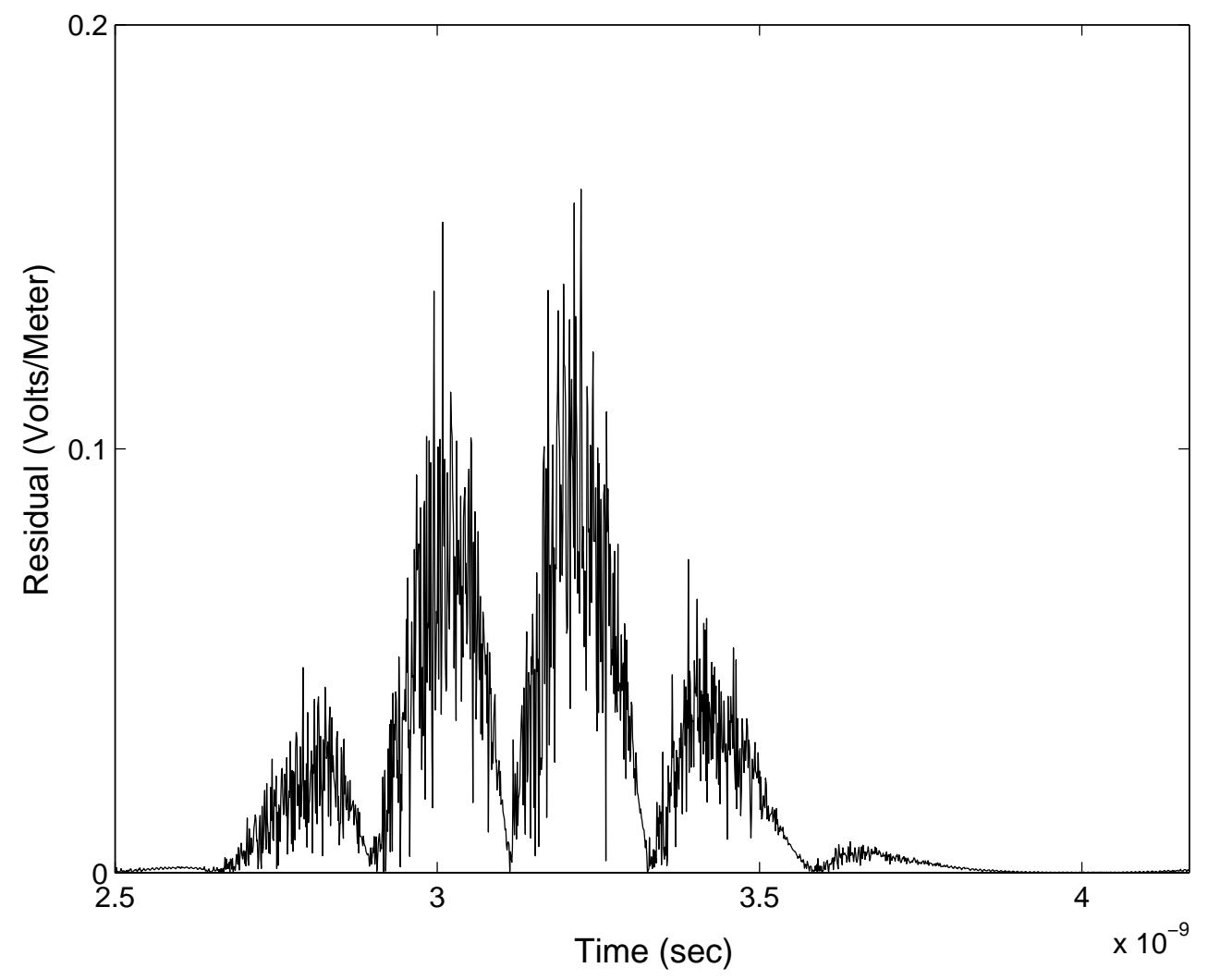

FIG. 10. A magnified plot of the least squares residual versus time in the interval where the acoustic reflection is observed, for the case of $10 \%$ relative random noise added to the data.

the simulated data with added relative random noise and added constant variance noise in Figures 14 17. We notice in Figure 17 that the acoustic reflection is drowned by the constant variance noise with $\nu=0.05(10 \% \mathrm{RN})$, which is not the case with the relative random noise data for the same value of $\nu$. Thus the identification of $\kappa_{s}$ is more difficult with constant variance noise added to the simulated data.

We next attempt the identification of three parameters, namely $\epsilon_{s, 0}, \tau_{0}$ and $\kappa_{s}$. The other four parameters, $\epsilon_{\infty, 0}, \sigma, \kappa_{\infty}$ and $\kappa_{\tau}$ are fixed at values given in (80). Tables 8 and 9 present the final estimates and the details of the corresponding Nelder-Mead simulation. We observe that the final estimates for both $\tau_{0}$ and $\kappa_{s}$ start to increase away from their true values as the level of constant variance noise is increased. The plots of the least squares objective function presented before provide an explanation for this.

In the case of the estimation of three parameters we observe that the estimates of $\kappa_{s}$ are more accurate when $\tau_{0}$ is allowed to vary as opposed to the case of two parameter estimation when $\tau_{0}$ is fixed at a particular value. Varying $\tau_{0}$, however, does not seem to make much difference in estimating $\epsilon_{s, 0}$. Also, comparing the intervals in Tables 7 and 10 we find that we obtain tighter confidence intervals for the three parameter case as 


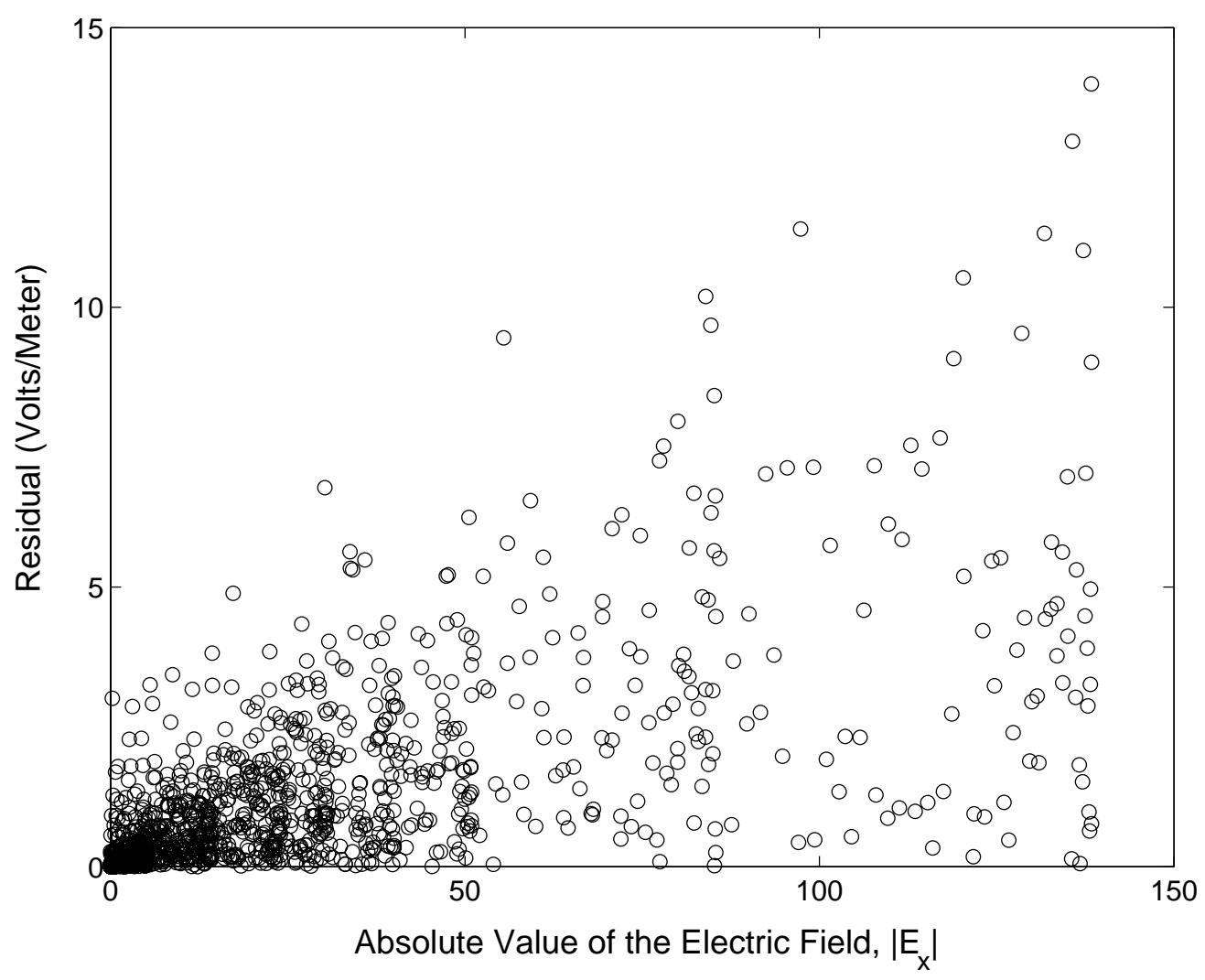

Fig. 11. Plot of the least squares residual versus the absolute value of the electric field data for the case of $10 \%$ relative random noise added to the data.

TABle 8. Parameter estimation of $\epsilon_{s, 0}$ and $\tau_{0}$ and $\kappa_{s}$ for constant variance noise, corresponding to varying levels of relative noise with the Nelder-Mead algorithm.

\begin{tabular}{|c|c|c|c|c|}
\hline \% RN & Iter & $\hat{\epsilon}_{s, 0}$ & $\hat{\tau}_{0}\left(\times 10^{-12}\right)$ & $\hat{\kappa}_{s}$ \\
\hline 0.0 & 78 & 80.1069 & 8.1543 & 48.2026 \\
1.0 & 90 & 80.0745 & 8.2242 & 49.8265 \\
5.0 & 99 & 79.9315 & 8.5015 & 56.7926 \\
10.0 & 83 & 79.7087 & 8.8299 & 66.2803 \\
\hline
\end{tabular}

opposed to the two parameter case, for the same level of added noise. The reason for this again is most likely the dependence of the acoustic reflections on the value of $\tau_{0}$. Thus, the algorithm for the inverse problem can choose $\tau_{0}$ appropriately so that the value of $\kappa_{s}$ is minimized. As before, estimates for all three parameters become worse as the level of noise added is increased and the corresponding confidence intervals become larger as seen in Table 10, 


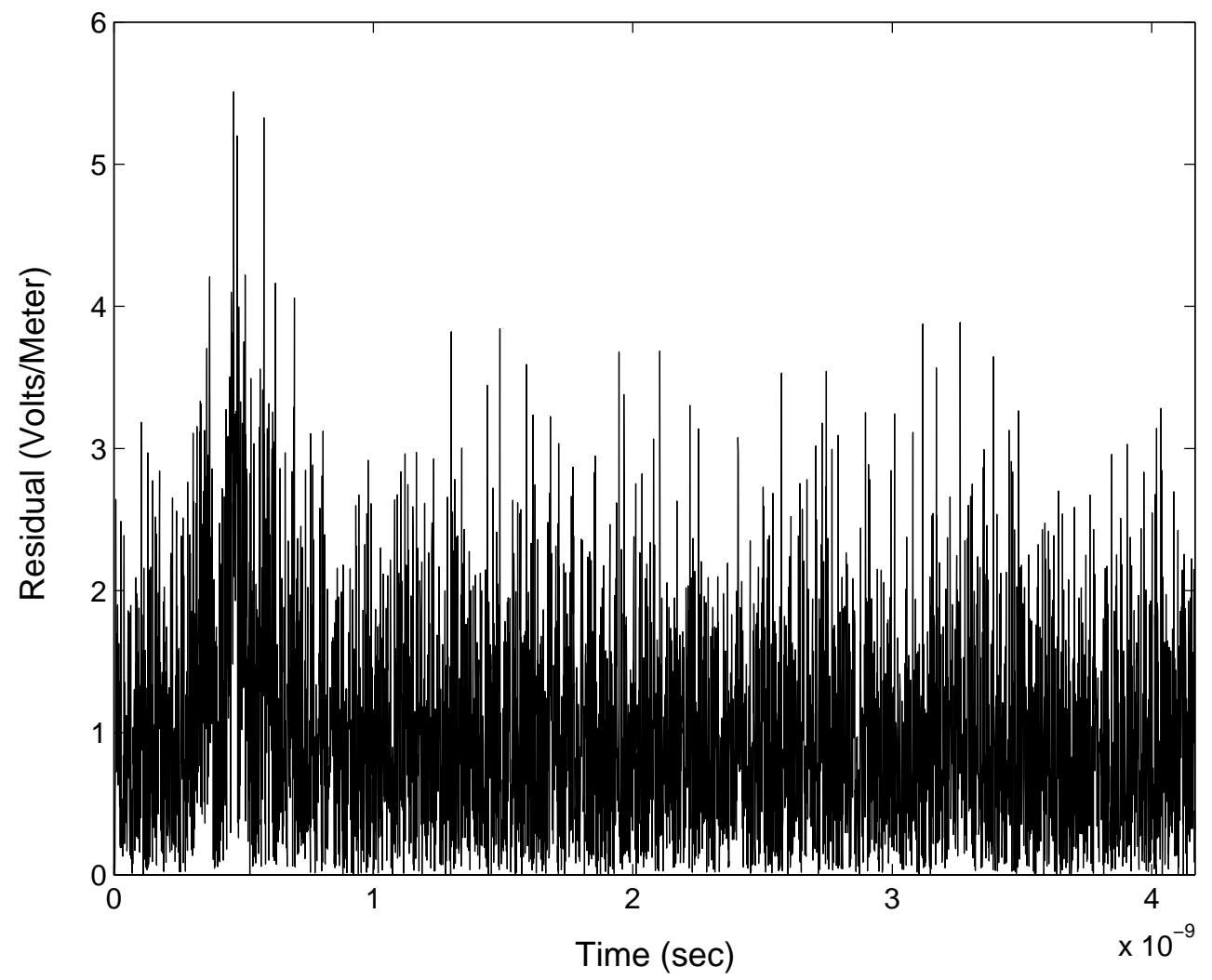

FIG. 12. Plot of the least squares residual versus time for the case of constant variance noise added to the simulated data.

TABle 9. Parameter estimation of $\epsilon_{s, 0}$ and $\tau_{0}$ and $\kappa_{s}$ for constant variance noise, corresponding to varying levels of relative noise with the Nelder-Mead algorithm.

\begin{tabular}{|c|c|c|c|l|}
\hline$\% \mathrm{RN}$ & $\left\|\nabla \mathrm{J}^{S}\right\|_{L^{2}}$ & $\mathrm{FC}$ & $\left|\mathrm{J}_{n+1}^{S}-\mathrm{J}_{0}^{S}\right|$ & $\mathrm{J}^{S}$ \\
\hline 0.0 & 0.008942 & 140 & $8.02867 \times 10^{-9}$ & $4.4391 \times 10^{-5}$ \\
1.0 & 0.001414 & 161 & $8.5522 \times 10^{-9}$ & 26.7071 \\
5.0 & 0.007697 & 181 & $6.7874 \times 10^{-9}$ & 667.6441 \\
10.0 & 0.001432 & 154 & $6.2983 \times 10^{-9}$ & 2670.5056 \\
\hline
\end{tabular}

6.3. Simulation Results: The Levenberg-Marquardt method. We now repeat our inverse problem calculations with a different least squares optimization technique. The NelderMead method presented in the previous section is a gradient free method based on the use of simplices. We will now use a gradient based method, namely the Levenberg-Marquardt method, to solve our inverse problem. For the overdetermined least squares objective function

$$
\mathrm{J}^{S}(\mathbf{q})=\frac{1}{2} \sum_{k=1}^{M}\left|E_{x}\left(t_{k}, x_{c}, z_{c} ; \mathbf{q}\right)-O_{k}^{s}\right|^{2}=\frac{1}{2} R(\mathbf{q})^{T} R(\mathbf{q}),
$$




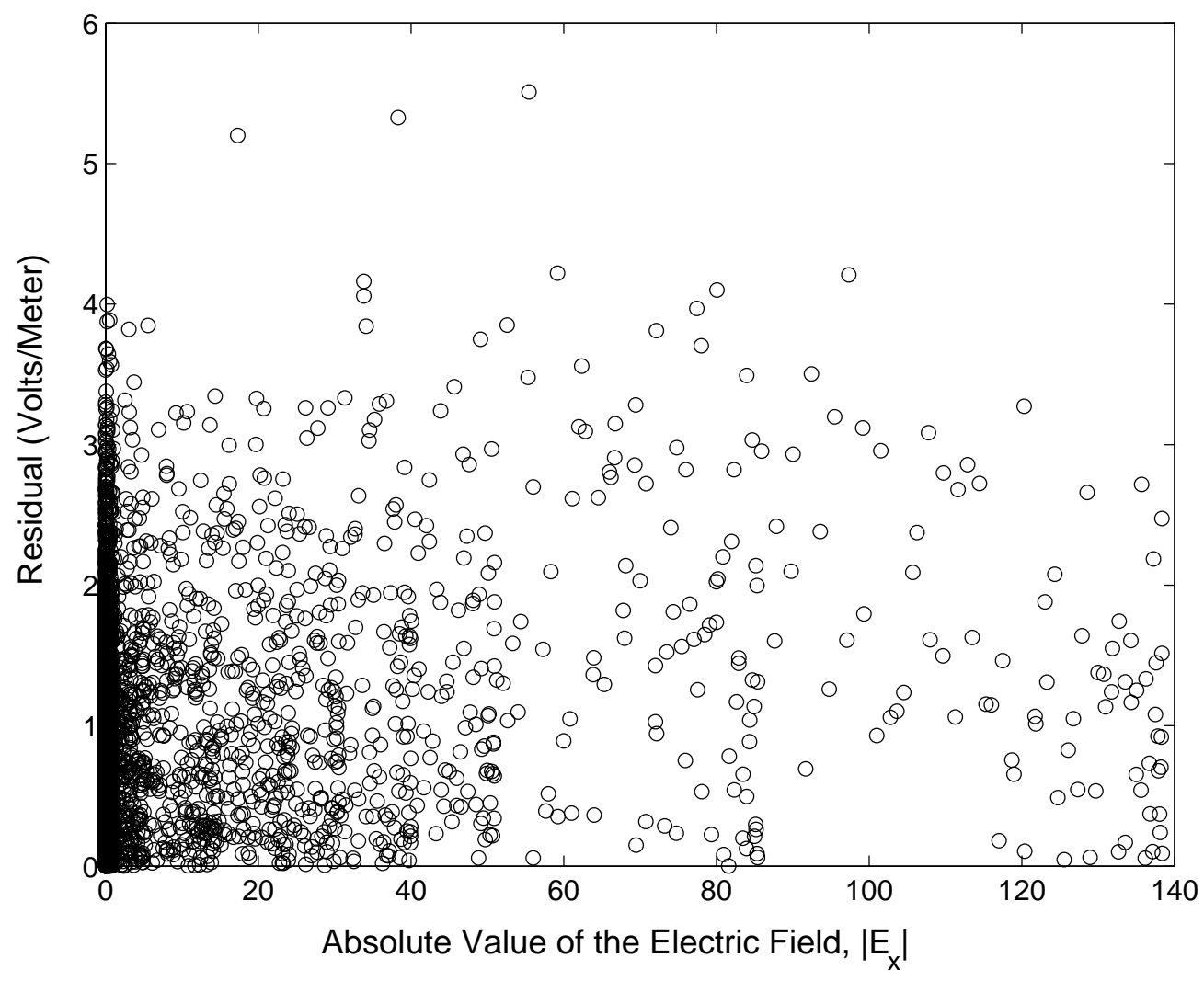

Fig. 13. Plot of the least squares residual versus the absolute value of the electric field for the case of constant variance noise added to the simulated data.

Levenberg-Marquardt, which is a trust region method, adds a regularization parameter $\nu_{L M}>0$ to the approximate Hessian of the Gauss-Newton method to obtain a new estimate $\mathbf{q}_{c}=\mathbf{q}_{c}+\mathbf{s}$, where

$$
\mathbf{s}=-\left(\nu_{L M} I+R^{\prime}\left(\mathbf{q}_{c}\right)^{T} R^{\prime}\left(\mathbf{q}_{c}\right)\right)^{-1} R^{\prime}\left(\mathbf{q}_{c}\right)^{T} R\left(\mathbf{q}_{c}\right),
$$

with $\mathbf{q}_{c}$ the current estimate and $I$ the $l \times l$ identity matrix, where $l$ is the number of parameters that we are attempting to estimate. The matrix $\left(\nu_{L M} I+R^{\prime}\left(\mathbf{q}_{c}\right)^{T} R^{\prime}\left(\mathbf{q}_{c}\right)\right)$ is positive definite. The parameter $\nu_{L M}$ is called the Levenberg-Marquardt parameter. For details of this implementation of the Levenberg-Marquardt method, we refer the reader to Kel99].

As with Nelder-Mead, we first attempt the identification of all the seven parameters, then we identify $\epsilon_{s, 0}$ and $\kappa_{s}$ and finally we attempt the identification of $\epsilon_{s, 0}, \tau_{0}$ and $\kappa_{s}$.

Table 11 presents the results for the inverse problem of identifying the seven parameters. We observe how close the final estimates of the parameters here are with those computed by Nelder-Mead. We can again make conclusions similar to those for the 


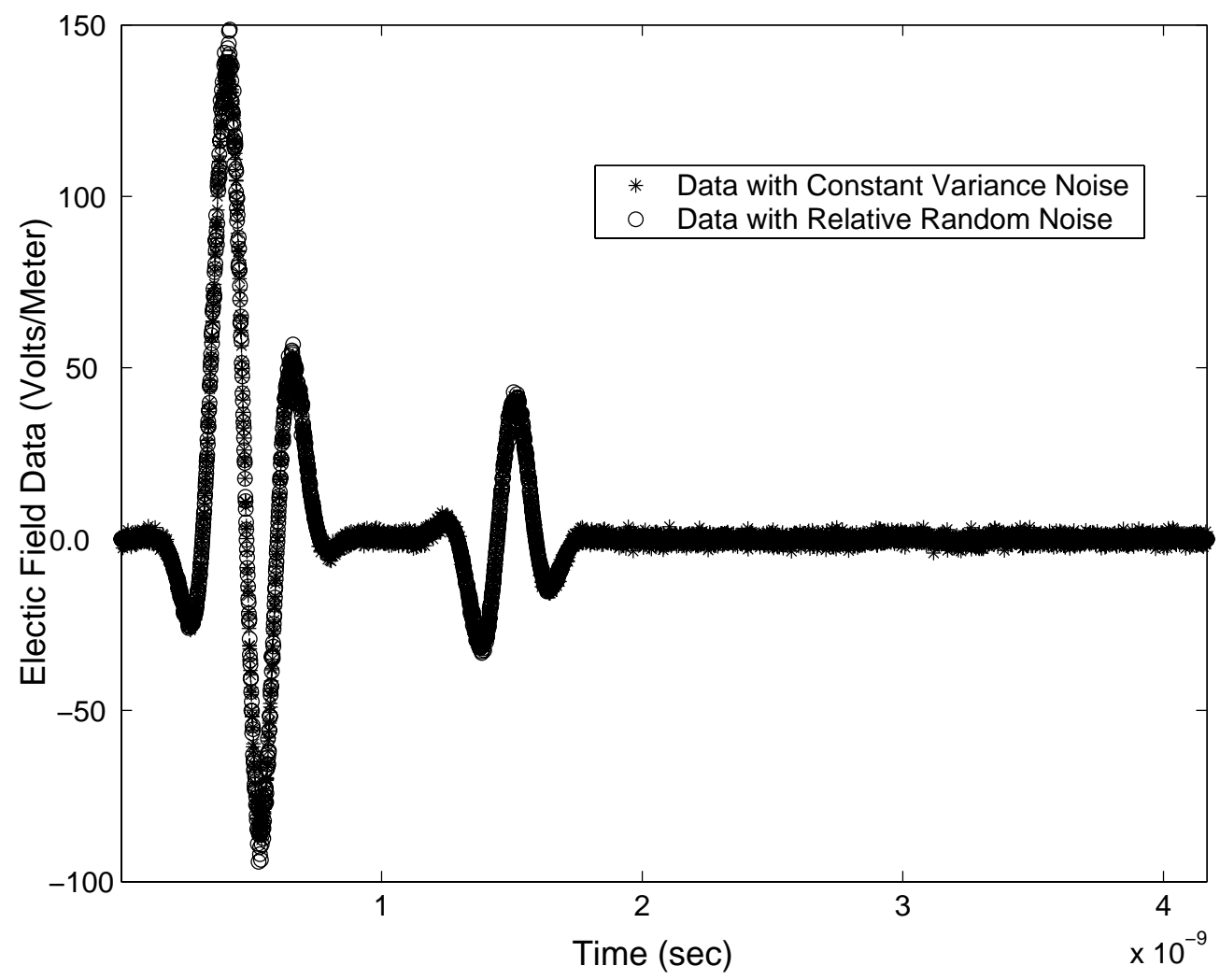

FIG. 14. Comparison of the data with added relative random noise to the data with constant variance noise added. The value of $\nu$ in both the cases is $\nu=0.05(10 \% \mathrm{RN})$.

Nelder-Mead results. Only $\epsilon_{s, 0}, \tau_{0}$ and $\kappa_{s}$ seem to be identifiable with some accuracy, whereas the problem appears to be insensitive to changes in the other four parameters.

We next attempt the identification of $\epsilon_{s, 0}$ and $\kappa_{s}$ with simulated data in which constant variance noise is added. The values of the remaining five parameters are fixed at those in (80). Table 12 presents the final estimates and the details of the corresponding LM simulation. The confidence intervals for these estimates are presented in Table13. Again, we can make remarks and observations similar to those for results of the Nelder-Mead method.

Finally we attempt to identify the three parameters $\epsilon_{s, 0}, \tau_{0}$ and $\kappa_{s}$. Table 14 presents the final estimates and details of the algorithm. Again, as was observed in the results of the Nelder-Mead runs, the estimates for $\kappa_{s}$ are better when the value of $\tau_{0}$ is kept variable as opposed to fixing the value of $\tau_{0}$.

In Table 15 we present the confidence intervals for the three parameter estimation problem. 
TABLE 10. Confidence intervals for the parameter estimation of $\epsilon_{s, 0}, \tau_{0}$ and $\kappa_{s}$ for constant variance noise with the Nelder-Mead algorithm.

\begin{tabular}{|c|c|}
\hline $\mathrm{RN}$ & $0.0 \%$ \\
\hline$\hat{\epsilon}_{s, 0}$ & $\left(8.01069 \pm 1.8738 \times 10^{-5}\right) \times 10$ \\
$\hat{\tau}_{0}$ & $\left(8.1543 \pm 2.3240 \times 10^{-4}\right) \times 10^{-12}$ \\
$\hat{\kappa}_{s}$ & $\left(4.82026 \pm 2.6832 \times 10^{-4}\right) \times 10$ \\
\hline $\mathrm{RN}$ & $1.0 \%$ \\
\hline$\hat{\epsilon}_{s, 0}$ & $\left(8.00745 \pm 1.4327 \times 10^{-2}\right) \times 10$ \\
$\hat{\tau}_{0}$ & $\left(8.2242 \pm 1.7739 \times 10^{-1}\right) \times 10^{-12}$ \\
$\hat{\kappa}_{s}$ & $\left(4.98265 \pm 2.0555 \times 10^{-1}\right) \times 10$ \\
\hline $\mathrm{RN}$ & $5.0 \%$ \\
\hline$\hat{\epsilon}_{s, 0}$ & $\left(7.99315 \pm 6.7761 \times 10^{-2}\right) \times 10$ \\
$\hat{\tau}_{0}$ & $\left(8.5015 \pm 8.3067 \times 10^{-1}\right) \times 10^{-12}$ \\
$\hat{\kappa}_{s}$ & $\left(5.67926 \pm 9.7751 \times 10^{-1}\right) \times 10$ \\
\hline $\mathrm{RN}$ & $10.0 \%$ \\
\hline$\hat{\epsilon}_{s, 0}$ & $\left(7.97087 \pm 1.2738 \times 10^{-1}\right) \times 10$ \\
$\hat{\tau}_{0}$ & $(8.8299 \pm 1.5286) \times 10^{-12}$ \\
$\hat{\kappa}_{s}$ & $(6.62803 \pm 1.8366) \times 10$ \\
\hline
\end{tabular}

TABLE 11. Final estimates for all seven parameters for water using Levenberg-Marquardt. The initial parameter set has values that are $5 \%$ smaller than the true values.

\begin{tabular}{|c|c|c|}
\hline Parameter & True Values & Final Estimates \\
\hline$\epsilon_{s, 0}$ & 80.1 & 80.0917 \\
$\epsilon_{\infty, 0}$ & 5.5 & 5.2039 \\
$\tau_{0}$ & $8.1 e-12$ & $8.0666 \times 10^{-12}$ \\
$\sigma$ & $1.0 \times 10^{-5}$ & $9.5 \times 10^{-6}$ \\
$\hat{\kappa}_{s}$ & 48.06 & 48.0028 \\
$\kappa_{\infty}$ & 4.4 & 4.1786 \\
$\kappa_{\tau}$ & $4.05 \times 10^{-13}$ & $3.8462 \times 10^{-13}$ \\
\hline
\end{tabular}

TABle 12. Parameter estimation of $\epsilon_{s, 0}$ and $\kappa_{s}$ for constant variance noise, corresponding to varying levels of relative noise with the Levenberg-Marquardt algorithm.

\begin{tabular}{|c|c|c|c|c|c|}
\hline$\%$ RN & Iter & $\hat{\epsilon}_{s, 0}$ & $\hat{\kappa}_{s}$ & $\mathrm{~J}^{S}$ & $\left\|\nabla \mathrm{J}^{S}\right\|_{L^{2}}$ \\
\hline 0.0 & 18 & 80.2166 & 55.2951 & 0.4369 & 0.0006526 \\
1.0 & 18 & 80.1654 & 56.4524 & 27.0778 & 0.00076 \\
5.0 & 18 & 79.9621 & 61.1765 & 667.7927 & 0.001385 \\
10.0 & 19 & 79.7084 & 67.3217 & 2670.5115 & 0.001417 \\
\hline
\end{tabular}




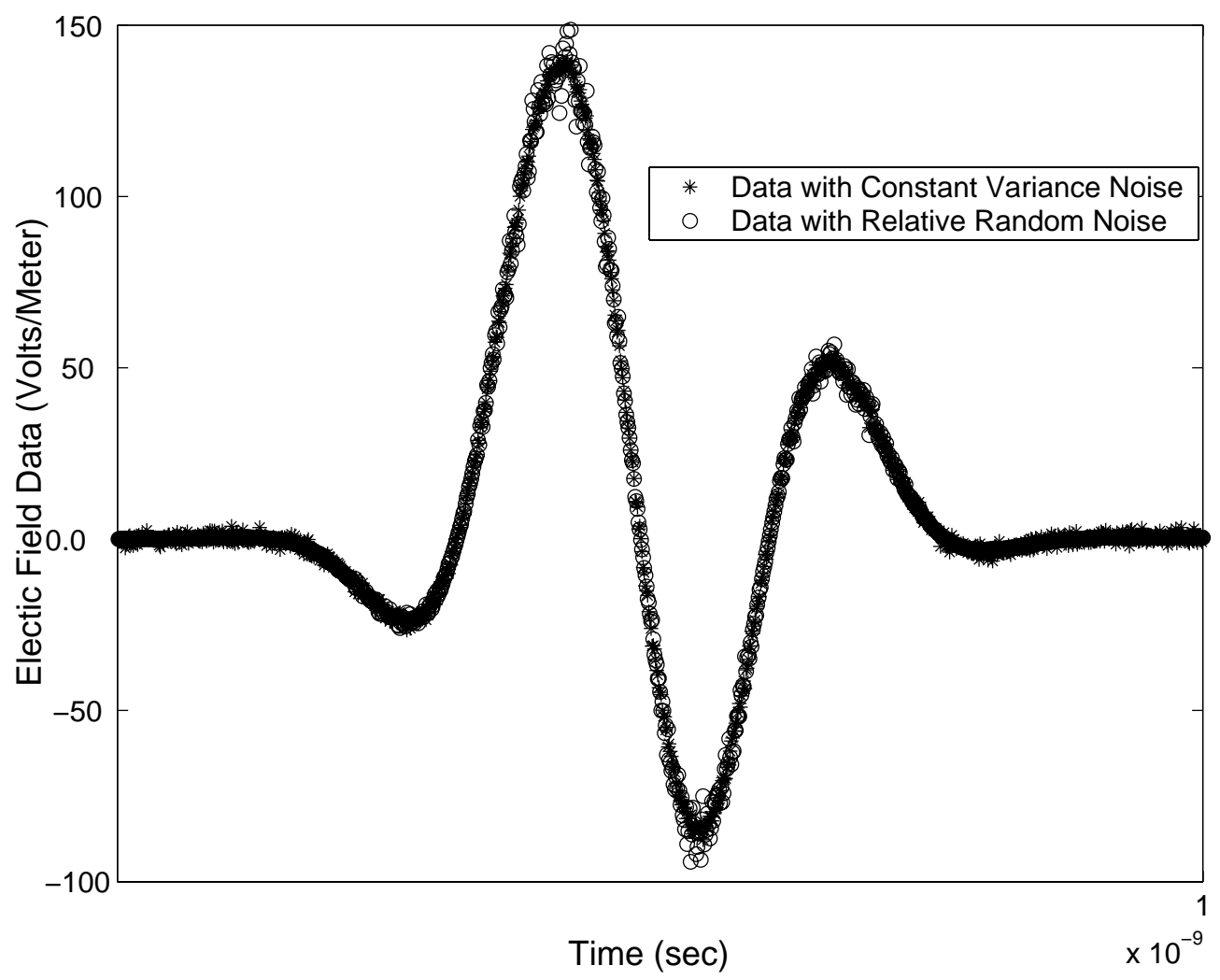

FIG. 15. Comparison of the data with added relative random noise to the data with constant variance noise added for the input signal at the center of the antenna. The value of $\nu$ in both the cases is $\nu=0.05(10 \% \mathrm{RN})$.

TABLE 13. Confidence intervals for the parameter estimation of $\epsilon_{s, 0}$ and $\kappa_{s}$ for constant variance noise with the Levenberg-Marquardt algorithm.

\begin{tabular}{|c|c|}
\hline & $\mathrm{RN}=0.0 \%$ \\
\hline$\hat{\epsilon}_{s, 0}$ & $\left(8.02166 \pm 1.8804 \times 10^{-3}\right) \times 10$ \\
$\hat{\kappa}_{s}$ & $\left(5.52951 \pm 1.6448 \times 10^{-2}\right) \times 10$ \\
\hline & $\mathrm{RN}=1.0 \%$ \\
\hline$\hat{\epsilon}_{s, 0}$ & $\left(8.01654 \pm 1.4577 \times 10^{-2}\right) \times 10$ \\
$\hat{\kappa}_{s}$ & $\left(5.64524 \pm 1.2738 \times 10^{-1}\right) \times 10$ \\
\hline & $\mathrm{RN}=5.0 \%$ \\
\hline$\hat{\epsilon}_{s, 0}$ & $\left(7.99621 \pm 6.8206 \times 10^{-2}\right) \times 10$ \\
$\hat{\kappa}_{s}$ & $\left(6.11765 \pm 5.9447 \times 10^{-1}\right) \times 10$ \\
\hline & $\mathrm{RN}=10.0 \%$ \\
\hline$\hat{\epsilon}_{s, 0}$ & $\left(7.97084 \pm 1.2723 \times 10^{-1}\right) \times 10$ \\
$\hat{\kappa}_{s}$ & $(6.73217 \pm 1.1076) \times 10$ \\
\hline
\end{tabular}




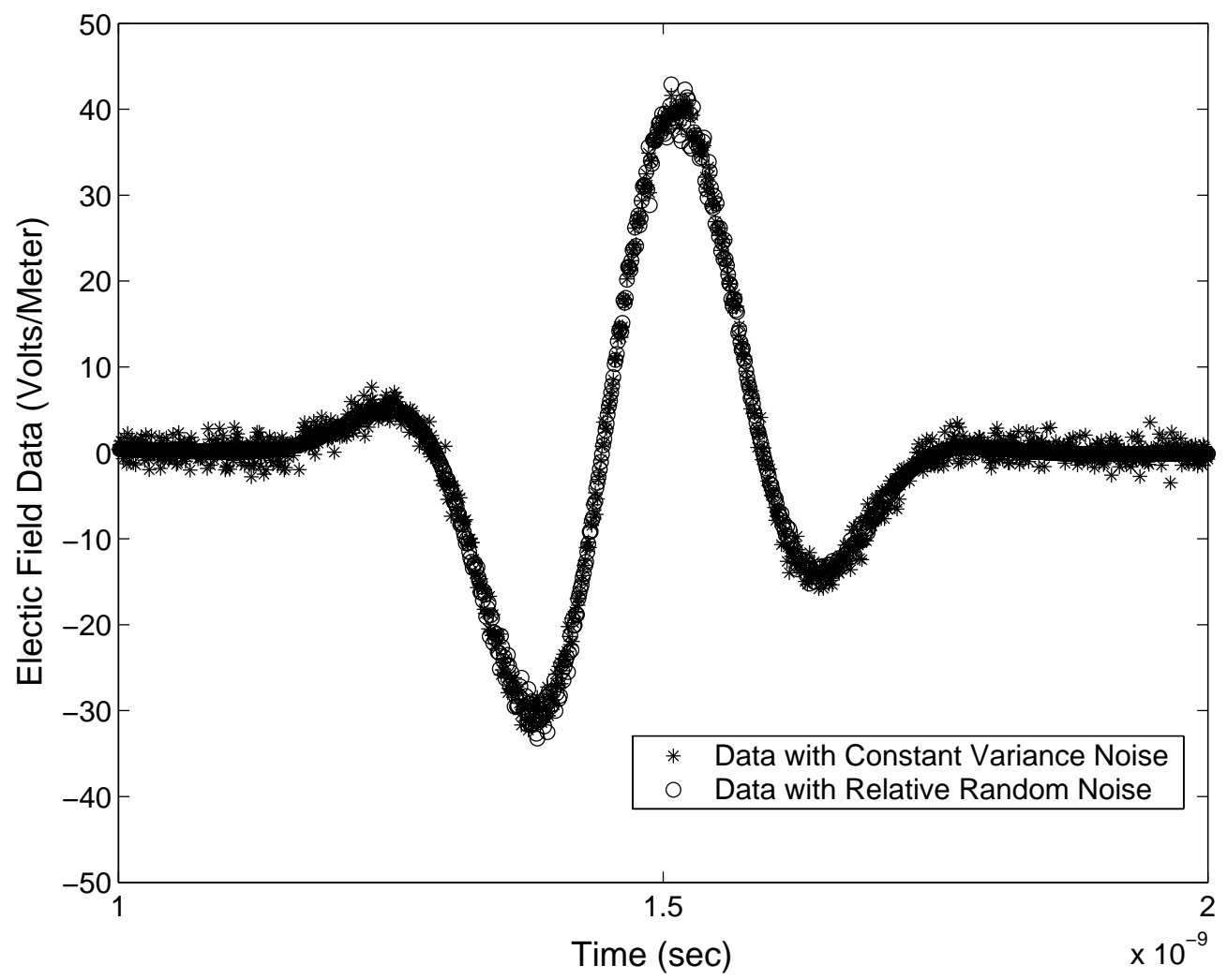

FIG. 16. Comparison of the data with added relative random noise to the data with constant variance noise added for the first reflection from the air-Debye interface. The value of $\nu$ in both the cases is $\nu=0.05(10 \% \mathrm{RN})$.

TABle 14. Parameter estimation of $\epsilon_{s, 0}$ and $\tau_{0}$ and $\kappa_{s}$ for constant variance noise, corresponding to varying levels of relative noise with the Levenberg-Marquardt algorithm.

\begin{tabular}{|c|c|c|c|c|c|c|}
\hline$\% \mathrm{RN}$ & Iter & $\hat{\epsilon}_{s, 0}$ & $\hat{\tau}_{0}\left(\times 10^{-12}\right)$ & $\hat{\kappa}_{s}$ & $\mathrm{~J}^{S}$ & $\left\|\nabla \mathrm{J}^{S}\right\|_{L^{2}}$ \\
\hline 0.0 & 17 & 80.1083 & 8.1562 & 48.2113 & $4.4383 \times 10^{-5}$ & 0.0009637 \\
1.0 & 17 & 80.0765 & 8.2247 & 49.8060 & 26.7071 & 0.001371 \\
5.0 & 19 & 79.9320 & 8.4983 & 56.6654 & 667.6441 & 0.0008044 \\
10.0 & 20 & 79.7085 & 8.8330 & 66.3476 & 2670.5056 & 0.001268 \\
\hline
\end{tabular}

7. Sensitivity of the parameter estimation problem to the relaxation constant and the interrogating frequency. In [BB04b] we have also considered a second parameter estimation test problem with a different interrogating frequency for a Debye medium with a relaxation time larger than that considered in the first test problem. This was done in order to study the effect of the relaxation time $\tau$ and the interrogation frequency $\omega_{c}$ on the ability of electromagnetic pulse to penetrate the dielectric material. As 


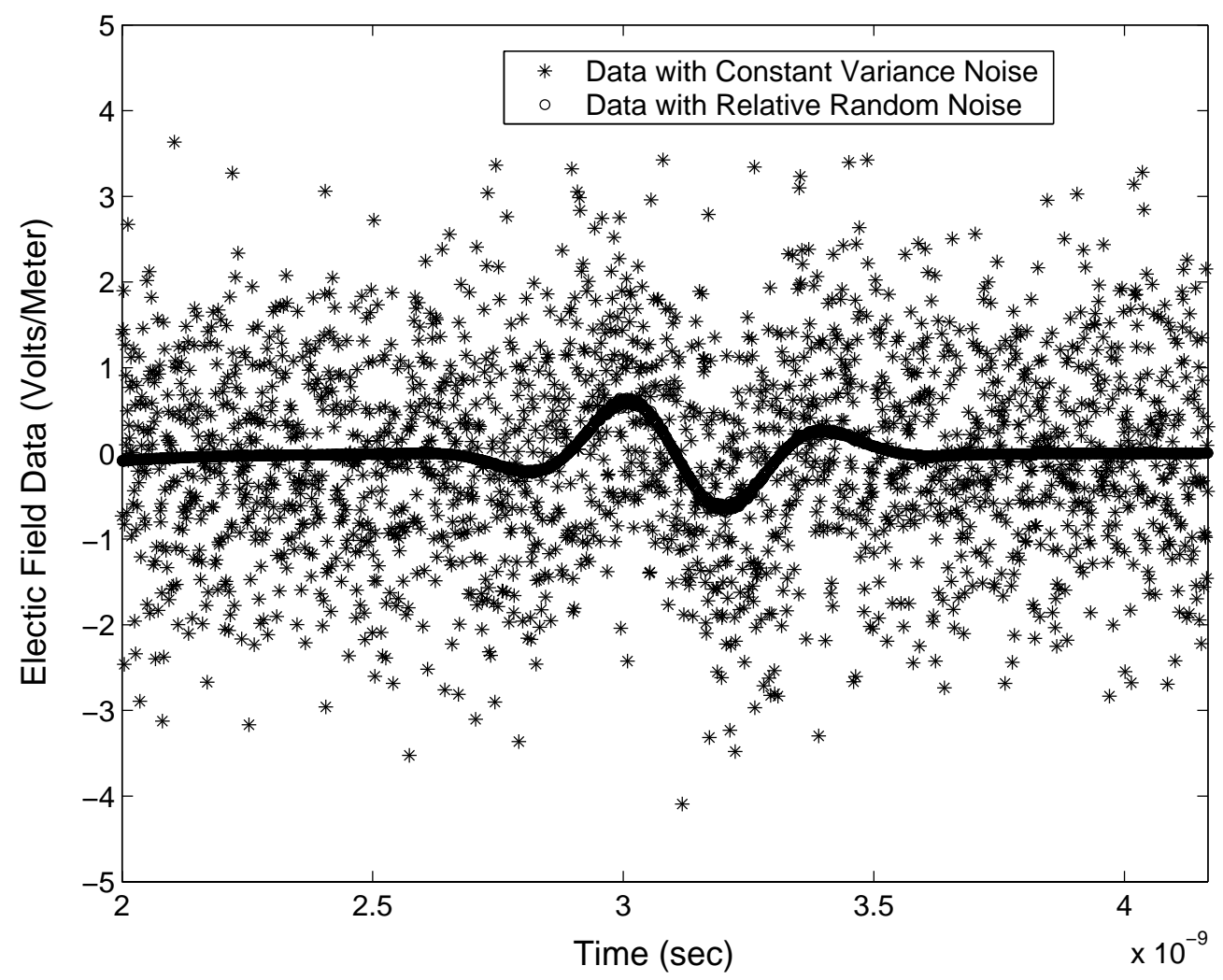

FIG. 17. Comparison of the data with added relative random noise to the data with constant variance noise added for the reflection from the acoustic wave region. The value of $\nu$ in both the cases is $\nu=0.05$ $(10 \% \mathrm{RN})$.

shown in [BBL00] a very small relaxation time, such as that for water, $\tau_{0}^{*}=8.1 \times 10^{-12}$, will make the material appear comparatively "hard" in the sense that it will allow much less of the signal to penetrate the air-Debye interface at $z=z_{1}$ for a sufficiently high interrogation frequency like $\omega_{c}=\pi \times 10^{10} \mathrm{rad} / \mathrm{sec}$. This small transmitted wave can only generate very little, if any, reflection at the second interface as it enters the region containing the pressure wave. This demonstrates the importance of the choice of the carrier frequency $\omega_{c}$ for effective interrogation. In [BBL00] the authors have demonstrated the strong influence of the dielectric relaxation time on an appropriate choice of the interrogating frequency for successfully penetrating the material. In our second test example (reported on in detail in [BB04b]) we considered a parameter estimation problem for a Debye medium with the relaxation time $\tau_{0}^{*}=3.16 \times 10^{-8}$ (as used in [BBL00, ABR02]), and an interrogation frequency $\omega_{c}=\pi \times 10^{10} \mathrm{rad} / \mathrm{sec}$.

As observed in Section 4.1, the product $\omega_{c} \tau_{0}$ strongly determines the parameters in the problem that can be identified accurately. We observe that for the choices of values of $\tau_{0}$ and $\omega_{c}$ in this second test problem, the parameters that dominate in the term $\epsilon_{r}^{*}$ in (51) 
TABLE 15. Confidence intervals for the parameter estimation of $\epsilon_{s, 0}$, $\tau_{0}$ and $\kappa_{s}$ for constant variance noise with the Levenberg-Marquardt algorithm.

\begin{tabular}{|c|c|}
\hline $\mathrm{RN}$ & $0.0 \%$ \\
\hline$\hat{\epsilon}_{s, 0}$ & $\left(8.01083 \pm 1.8741 \times 10^{-5}\right) \times 10$ \\
$\hat{\tau}_{0}$ & $\left(8.1562 \pm 2.3234 \times 10^{-4}\right) \times 10^{-12}$ \\
$\hat{\kappa}_{s}$ & $\left(4.82113 \pm 2.6830 \times 10^{-4}\right) \times 10$ \\
\hline $\mathrm{RN}$ & $1.0 \%$ \\
\hline$\hat{\epsilon}_{s, 0}$ & $\left(8.00765 \pm 1.4334 \times 10^{-2}\right) \times 10$ \\
$\hat{\tau}_{0}$ & $\left(8.2247 \pm 1.7742 \times 10^{-1}\right) \times 10^{-12}$ \\
$\hat{\kappa}_{s}$ & $\left(4.98060 \pm 2.0560 \times 10^{-1}\right) \times 10$ \\
\hline $\mathrm{RN}$ & $5.0 \%$ \\
\hline$\hat{\epsilon}_{s, 0}$ & $\left(7.99320 \pm 6.7841 \times 10^{-2}\right) \times 10$ \\
$\hat{\tau}_{0}$ & $\left(8.4983 \pm 8.3159 \times 10^{-1}\right) \times 10^{-12}$ \\
$\hat{\kappa}_{s}$ & $\left(5.66654 \pm 9.7843 \times 10^{-1}\right) \times 10$ \\
\hline $\mathrm{RN}$ & $10.0 \%$ \\
\hline$\hat{\epsilon}_{s, 0}$ & $\left(7.97085 \pm 1.2736 \times 10^{-1}\right) \times 10$ \\
$\hat{\tau}_{0}$ & $(8.8330 \pm 1.5277) \times 10^{-12}$ \\
$\hat{\kappa}_{s}$ & $(6.63476 \pm 1.8360) \times 10$ \\
\hline
\end{tabular}

are no longer the same as those of the first test problem, i.e., $\epsilon_{s, 0}$ and $\kappa_{s}$. The parameters whose magnitude dominates the quantity $\epsilon_{r}^{*}$ are $\epsilon_{\infty, 0}$ and the pressure coefficient $\kappa_{\infty}$. This observation, as was done for the first test problem, can also be deduced from an analysis of (31). In the simulations for this test problem the outgoing and reflected radiation will be dominated by frequencies near the center frequency $5.0 \mathrm{GHz}$. Thus, $\epsilon_{r}^{*}$ will be dominated by frequencies near $5.0 \mathrm{GHz}$. In this problem $\omega \tau_{0}^{*} \approx \mathcal{O}\left(10^{2}\right)$, and with $\epsilon_{0}=8 \times 10^{-12}$ we have (compare with the estimates of (152)

$$
\begin{aligned}
& \left(\frac{\epsilon_{s, 0}^{*}}{1+\mathrm{j} \omega \tau_{0}^{*}}\right) \quad \approx \mathcal{O}\left(10^{-2} \epsilon_{s, 0}^{*}\right) \approx \mathcal{O}\left(10^{-1}\right), \\
& \left(\frac{\mathrm{j} \omega \tau_{0}^{*}}{1+\mathrm{j} \omega \tau_{0}^{*}}\right) \epsilon_{\infty, 0}^{*} \approx \mathcal{O}\left(\epsilon_{\infty, 0}^{*}\right) \quad \approx \mathcal{O}(10), \\
& \left(\frac{\sigma^{*}}{\mathrm{j} \omega \epsilon_{0}}\right) \quad \approx \mathcal{O}\left(10^{3} \sigma^{*}\right) \quad \approx \mathcal{O}\left(10^{-3}\right) .
\end{aligned}
$$

Thus we can see that $\epsilon_{r}^{*}$ will be most sensitive to the infinite frequency permittivity $\epsilon_{\infty, 0}$ and the effects of $\epsilon_{s, 0}$ and $\tau_{0}$ and $\sigma$ will not be as pronounced. Also, this implies that $\epsilon_{r}^{*}$ will be more sensitive to the pressure coefficient $\kappa_{\infty}$ than to the coefficients $\kappa_{s}$ and $\kappa_{\tau}$.

As shown in [BB04b], this second parameter estimation problem is very different than the one that we have considered in this paper. We summarize the essential differences between these two problems below.

(1) The parameters that can be accurately estimated in the two test cases are different. In the first example of the Debye medium that is similar to water we found that $\epsilon_{s, 0}$ and the corresponding pressure coefficient $\kappa_{s}$ can be accurately identified. In the second example, however, it is $\epsilon_{\infty, 0}$ and the corresponding 
pressure coefficient $\kappa_{\infty}$ that can be accurately identified. The parameters that can be identified in an inverse problem clearly depend on the magnitude of the product $\omega_{c} \tau_{0}$.

(2) In the case of the second test problem, which has a larger relaxation time, we see presence of local minima in the objective function. The distance between the local minima increases as the interrogating frequency is decreased, since then the product $\omega_{c} \tau_{0}$ decreases. Thus, by choosing a lower interrogating frequency the local minima could be pushed out of the domain of interest. In contrast, we do not see the presence of local minima in the first parameter estimation problem which has a much smaller value of $\tau_{0}$.

(3) The behavior of the Nelder-Mead algorithm for the parameter estimation problem for the first Debye medium is markedly different than for the second example. In the example with local minima present, the Nelder-Mead algorithm converges to a local minimum instead of the global minimum. We do not see this behavior with the Levenberg-Marquardt algorithm for the same example. In contrast, in the parameter estimation problem for water, both Nelder-Mead as well as Levenberg-Marquardt converge to the global minimum and demonstrate similar behavior.

8. Conclusions and future directions. In this paper we have presented an electromagnetic interrogation technique for identifying dielectric parameters of a Debye medium by using acoustic pressure waves as virtual reflectors for the incident electromagnetic pulse. We considered the two dimensional TE mode of Maxwell's equations which incorporates the pressure dependence in the Debye medium via a model for orientational polarization. As a first approximation we assumed that the dielectric parameters $\epsilon_{s, 0}$, $\epsilon_{\infty, 0}$ and the relaxation $\tau_{0}$ are affine functions of pressure. We have used a finite difference time domain scheme to discretize our electromagnetic/acoustic model and to compute simulated data.

We formulated a least squares based inverse problem for the identification of the material parameters as well as the coefficients of pressure in the Debye model. We used two different methods to solve the inverse problem; namely Levenberg-Marquardt and Nelder-Mead. The inverse problem calculations indicate that the coefficients that can be identified depend on the order of magnitude of $\omega \tau_{0}$. Thus in the first test problem we were able to identify $\epsilon_{s, 0}$ and the corresponding pressure coefficient $\kappa_{s}$, and in the second test problem we were able to identify $\epsilon_{\infty, 0}$ and the corresponding pressure coefficient $\kappa_{\infty}$. It is not possible to accurately identify all the seven parameters that describe the Debye medium coupled with the model for pressure dependence in either of the examples that we have considered in this paper.

We have also computed confidence intervals for all the estimates obtained using statistical error analysis based on the assumption of constant variance noise. We demonstrated that the intervals become larger as the level of added noise is increased.

There are still many questions to be investigated. We have used a simple linear model for the pressure term in Maxwell's equations. Perhaps more importantly, we have assumed that the effect of the electromagnetic field on the acoustic wave is negligible. A 
dynamic coupled model of the electromagnetic/acoustic interaction is the topic of future efforts. We will also consider models in which the dielectric parameters are nonlinear functions of pressure in attempts to understand better the dynamical pressure dependence of the dielectric parameters.

9. Acknowledgements. This research was supported in part by the U.S. Air Force Office of Scientific Research under grants AF0SR F49620-01-1-0026 and AFOSR FA955004-1-0220. The authors would like to thank Dr. Richard Albanese of the AFRL, Brooks AFB, Brian Adams, Nathan Gibson, Dr. Grace Kepler, Dr. Yanyuan Ma, and Dr. Jari Toivanen of CRSC at NCSU for their valuable comments and suggestions.

\section{REFERENCES}

[ABK04] R. Albanese, H. T. Banks, and G. M. Kepler, Experimental results involving acousto-optical interactions, Tech. Report in preparation, CRSC, N. C. State University, 2004.

[ABR02] R. A. Albanese, H. T. Banks, and J. K. Raye, Nondestructive evaluation of materials using pulsed microwave interrogating signals and acoustic wave induced reflections, Inverse Problems 18 (2002), 1935-1958. MR1955927 (2003k:78021)

[AMP94] R. A. Albanese, R. L. Medina, and J. W. Penn, Mathematics, medicine and microwaves, Inverse Problems 10 (1994), 995-1007. MR.1296358

[And67] J. C. Anderson, Dielectrics, Chapman and Hall, London, 1967.

[APM89] R. A. Albanese, J. W. Penn, and R. L. Medina, Short-rise-time microwave pulse propagation through dispersive biological media, J. Optical Society of America A 6 (1989), 1441-1446.

[BB] H. T. Banks and K. L. Bihari, Modeling and estimating uncertainty in parameter estimation, Tech. Report CRSC-TR99-40, N. C. State University, Dec. 1999; Inverse Problems 17: 95-111, 2001. MR.1818494 (2002d:35215)

[BB04a] H. T. Banks and J. M. Bardsley, Parameter identification for a dispersive dielectric in $2 d$ electromagnetics: Forward and inverse methodology with statistical considerations, Tech. Report CRSC-TR03-48, N. C. State University, Dec. 2003; Intl. J. Comp. and Num. Anal. and Applic., to appear, 2004.

[BB04b] H. T. Banks and V. A. Bokil, Parameter identification for dispersive dielectrics using pulsed microwave interrogating signals and acoustic wave induced reflections in two and three dimensions, Tech. Report CRSC-TR04-27, N. C. State University, July 2004.

[BBL00] H. T. Banks, M. W. Buksas, and T. Lin, Electromagnetic material interrogation using conductive interfaces and acoustic wavefronts, Frontiers in Applied Mathematics, vol. FR21, SIAM, Philadelphia, PA, 2000. MR1787981|(2001k:78036)

[Ber94] J. P. Berenger, A perfectly matched layer for the absorption of electromagnetic waves, J. Comput. Phys. 114 (1994), no. 2, 185-200. MR.1294924|(95e:78002)

[BF95] J. G. Blaschak and J. Franzen, Precursor propagation in dispersive media from short-rise-time pulses at oblique incidence, J. Optical Society of America A 12 (1995), 1501-1512.

[BG04] H. T. Banks and N. L. Gibson, Well-posedness in maxwell systems with distributions of polarization relaxation parameters, Tech. Report CRSC-TR04-01, N. C. State University, Jan. 2004; Applied Math. Letters, to appear, 2004.

[BGW03] H. T. Banks, N. L. Gibson, and W. P. Winfree, Electromagnetic crack detection inverse problems using terahertz interrogating propagating signals, Tech. Report CRSC-TR03-40, N. C. State University, October 2003.

[BK89] H. T. Banks and K. Kunisch, Estimation techniques for distributed parameter systems, Birkhauser, Boston, 1989. MR,1045629 (91b:93085)

[Bri60] L. Brillouin, Wave propagation and group velocity, Academic Press, New York and London, 1960. MR0108217|(21:6933)

[DG95] M. Davidian and D. M. Giltinan, Nonlinear models for repeated measurement data, Monographs on Statistics and Applied Probability, vol. 62, Chapman and Hall, New York, 1995.

[FMS03] E. C. Fear, P. M. Meaney, and M. A. Stuchly, Microwaves for breast cancer detection, IEEE Potentials (2003), 12-18. 
[Ged96] S. D. Gedney, An anisotropic perfectly matched layer absorbing media for the truncation of FDTD lattices, IEEE Trans. Antennas Propagat. 44 (1996), no. 12, 1630-1639.

[Jac99] J. D. Jackson, Classical electromagnetics, John Wiley and Sons, New York, 1999.

[JN84] N. S. Jayant and P. Noll, Digital coding of waveforms, Prentice Hall, Englewood Cliffs, 1984.

[Kel99] C. T. Kelley, Iterative methods for optimization, Frontiers in Applied Mathematics, vol. FR18, SIAM, Philadelphia, PA, 1999. MR1678201 (2000h:90003)

[Kor97] A. Korpel, Acousto-optics, 2nd ed., Marcel-Dekker, New York, 1997.

[MI68] P. Morse and K. Ingard, Theoretical acoustics, McGraw-Hill, New York, 1968.

[MP95] R. Mittra and U. Pekel, A new look at the perfectly matched layer (PML) concept for the reflectionless absorption of electromagnetic waves, IEEE Microwave Guided Wave Lett. 5 (1995), no. 3, 84-86.

[Pet94] P. Petropoulos, Stability and phase error analysis of FDTD in dispersive dielectrics, IEEE Trans. Antennas Propagat. 42 (1994), no. 1, 62-69.

[Rap95] C. M. Rappaport, Perfectly matched absorbing boundary conditions based on anisotropic lossy mapping of space, IEEE Microwave Guided Wave Lett. 5 (1995), no. 3, 90-92.

[SKLL95] Z. S. Sacks, D. M. Kinsland, R. Lee, and J. F. Lee, A perfectly matched anisotropic absorber for use as an absorbing boundary condition, IEEE Trans. Antennas Propagat. 43 (1995), 1460-1463.

[Taf95] A. Taflove, Computational electrodynamics: The finite-difference time-domain method, Artech House, Norwood, MA, 1995. MR 1338377 (96f:78001)

[Taf98] - Advances in computational electrodynamics: The finite-difference time-domain method, Artech House, Norwood, MA, 1998. MR1639352 (99c:78001) 